\title{
ON STANDARD NORM VARIETIES
}

\author{
NIKITA A. KARPENKO AND ALEXANDER S. MERKURJEV
}

\begin{abstract}
Let $p$ be a prime integer and $F$ a field of characteristic 0 . Let $X$ be the norm variety of a symbol in the Galois cohomology group $H^{n+1}\left(F, \mu_{p}^{\otimes n}\right.$ ) (for some $n \geq 1$ ), constructed in the proof of the Bloch-Kato conjecture. The main result of the paper affirms that the function field $F(X)$ has the following property: for any equidimensional variety $Y$, the change of field homomorphism $\mathrm{CH}(Y) \rightarrow \mathrm{CH}\left(Y_{F(X)}\right)$ of Chow groups with coefficients in integers localized at $p$ is surjective in codimensions $<(\operatorname{dim} X) /(p-1)$. One of the main ingredients of the proof is a computation of Chow groups of a (generalized) Rost motive (a variant of the main result not relying on this is given in Appendix). Another important ingredient is A-triviality of $X$, the property saying that the degree homomorphism on $\mathrm{CH}_{0}\left(X_{L}\right)$ is injective for any field extension $L / F$ with $X(L) \neq \emptyset$. The proof involves the theory of rational correspondences, due to Markus Rost, reviewed in Appendix.
\end{abstract}

\section{Contents}

1. Introduction

2. A-trivial varieties

3. Abstract Rost motives

4. Generic splitting varieties

5. A-triviality of standard norm varieties

5a. Retract rational varieties 11

5b. A-trivial varieties revisited 11

\begin{tabular}{lll}
\hline $5 c . \quad$ Symmetric powers & 12
\end{tabular}

5d. A-triviality of standard norm varieties 13

\begin{tabular}{lll}
\hline Appendix RC. Rational correspondences & 17
\end{tabular}

\begin{tabular}{lll}
\hline RC-I. & Integral correspondences & 17
\end{tabular}

\begin{tabular}{lll|}
\hline RC-II. & The cycle module $A_{0} \mid Y, M$ & 18
\end{tabular}

\begin{tabular}{lll}
\hline RC-III. The category of rational correspondences & 21
\end{tabular}

\begin{tabular}{|ll}
\hline \hline Appendix RM. Chow groups of Rost motives & 24 \\
\hline
\end{tabular}

$\begin{array}{lll}\text { RM-1. The binary motivg } & 24\end{array}$

$\begin{array}{lll}\text { RM-II. Symmetric powers } & 25\end{array}$

RM-III. Chow groups of Rost motives 26

\begin{tabular}{lll}
\hline Appendix SC. Special correspondences & 28
\end{tabular}

\begin{tabular}{lll}
\hline SC-I. & Rationality of Steenrod operations & 28
\end{tabular}

SC-II. Generators of Chow groups of Rost motives 34

References 36

Date: 4 January 2012.

Key words and phrases. Norm varieties, Chow groups and motives, Steenrod operations. Mathematical Subject Classification (2010): 14C25.

Supported by the Max-Planck-Institut für Mathematik in Bonn.

The work of the second author has been supported by the NSF grant DMS \#0652316. 


\section{INTRODUCTION}

Let $n$ be a positive integer and $p$ a prime integer. A smooth complete geometrically irreducible variety $X$ over a field $F$ of characteristic 0 is a p-generic splitting variety for a symbol $s \in H^{n+1}\left(F ; \mu_{p}^{\otimes n}\right)$ if $s$ vanishes over a field extension $K / F$ if and only if $X$ over $K$ has a closed point of degree prime to $p$. A norm variety of $s$ is a p-generic splitting variety of the smallest dimension $p^{n}-1$. Norm varieties played an important role in the proof of the Bloch-Kato conjecture (see [33]).

Let $Y$ be a smooth variety over $F$. Write $\mathrm{CH}^{i}(Y)$ for the Chow group with coefficients in the integers localized at $p$ and $\mathrm{CH}^{i}(Y)$ for the factor group of the Chow group $\mathrm{CH}^{i}(Y)$ modulo $p$-torsion elements and $p \mathrm{CH}^{i}(Y)$. In [34, Theorem 1.3], K. Zainoulline proved, using the Landweber-Novikov operations in algebraic cobordism theory, that every $Y$ and every norm variety $X$ of $s$ enjoy the following property: if $i<\left(p^{n}-1\right) /(p-1)$, every class $\alpha$ in $\mathrm{CH}^{i}\left(Y_{\bar{F}}\right)$, where $\bar{F}$ is an algebraic closure of $F$, such that $\alpha_{\bar{F}(X)}$ is $F(X)$-rational, is $F$-rational itself, i.e., $\alpha$ belongs to the image of the map $\mathrm{CH}^{i}(Y) \rightarrow \mathrm{CH}^{i}\left(Y_{\bar{F}}\right)$. This statement is in the spirit of the Main Tool Lemma of A. Vishik 30].

In the present paper we improve this result by showing that every cycle in the Chow group $\mathrm{CH}^{i}\left(Y_{F(X)}\right)$ is already defined over $F$, i.e, it comes from $\mathrm{CH}^{i}(Y)$. More precisely, we prove the following theorem (see Theorem 4.3 for a stronger statement and the proof):

Theorem 1.1. Let $F$ be a field of characteristic 0 and let $X$ be an A-trivial p-generic splitting variety of a symbol in $H^{n+1}\left(F, \mu_{p}^{\otimes n}\right)$. Then the change of field homomorphism $\mathrm{CH}^{i}(Y) \rightarrow \mathrm{CH}^{i}\left(Y_{F(X)}\right)$ is surjective if $i<\left(p^{n}-1\right) /(p-1)$ for any equidimensional (not necessarily smooth) variety $Y$ over $F$. Moreover, the bound $\left(p^{n}-1\right) /(p-1)$ is sharp.

The $A$-triviality property for $X$ means that the degree map deg: $\mathrm{CH}_{0}\left(X_{K}\right) \rightarrow \mathbb{Z}_{(p)}$ is an isomorphism (i.e., the kernel $A\left(X_{K}\right)$ of the degree map is trivial) for any field extension $K / F$ such that $X$ has a point over $K$. (We believe that the $A$-triviality condition should be also imposed in the statement of [34, Theorem 1.3].) Our proof of Theorem 1.1] is "elementary" in the sense that it does not use the algebraic cobordism theory.

In Section 5 we prove that the standard norm varieties (corresponding to nontrivial symbols) constructed in 28] are $A$-trivial, so that Theorem 1.1 can be applied to such varieties. In fact, we prove more (see Theorem 5.8 for a more explicit statement and the proof):

Theorem 1.2. Let $X$ be a standard norm variety of a nontrivial symbol over a field $F$ of characteristic 0 . Then for any field extension $K / F$, the degree map deg: $\mathrm{CH}_{0}\left(X_{K}\right) \rightarrow \mathbb{Z}_{(p)}$ is injective.

In the proof we use the theory of rational correspondences developed by M. Rost (unpublished). We review this theory in Appendix RC. Another ingredient of the proof, a computation of Chow groups of Rost motives, is presented in Appendix RM. A variant of the main theorem valid in any characteristic $\neq p$ and involving the Steenrod operations is given in Appendix $\mathrm{Sq}$.

In Sections 3 and 4 we develop a theory of (abstract) Rost motives. 
We use the following notation and conventions. The base field $F$ is of arbitrary characteristic if not specified otherwise (it is of characteristic $\neq p, p$ a fixed prime, most of the time, and of characteristic 0 in several places). An $F$-variety over a field $F$ is a separated scheme of finite type over $F$.

We fix a commutative unital ring $\Lambda$ and write $\mathrm{CH}=\mathrm{CH}_{*}$ for the Chow group with coefficients in $\Lambda$. For any integer $i$ and equidimensional $F$-variety $Y$, we write $\mathrm{CH}^{i}(Y)$ for the Chow group $\mathrm{CH}_{\operatorname{dim} Y-i}(Y)$.

Many events in the paper happen in the category of Chow motives (with coefficients in $\Lambda$, see [7]). A Chow motive is a pair $(X, \rho)$, where $X$ is a smooth complete variety over $F$ and $\rho$ is a projector (idempotent) in the endomorphism ring of the motive $M(X)$ of $X$. We say that a motive $M$ lives on $X$, if $M \simeq(X, \rho)$ for some $\rho$ as above.

ACKnowledgements. The authors thank Markus Rost for useful comments and suggestions.

\section{2. $A$-trivial Varieties}

Let $X$ be a smooth complete irreducible $F$-variety. Let $d$ be its dimension, and let $\rho$ be a fixed element of the Chow group $\mathrm{CH}^{d}(X \times X)$ (considered as a correspondence $X \rightsquigarrow X)$.

Lemma 2.1. The following two conditions on $\rho$ are equivalent:

(1) for any F-variety $Y$, the image of any $\alpha \in \mathrm{CH}(X \times Y)$ under the pull-back to $\mathrm{CH}\left(Y_{F(X)}\right)$ coincides with the image of $\alpha \circ \rho$;

(2) $\rho_{*}[\xi]=[\xi]$, where $\xi$ is the generic point of $X$ and $[\xi]$ is its class in the Chow group $\mathrm{CH}_{0}\left(X_{F(X)}\right)$.

Proof. $(2) \Rightarrow(1)$. The image of $\alpha$ is equal to $\alpha_{*}[\xi]$. In particular, the image of $\alpha \circ \rho$ is equal to $(\alpha \circ \rho)_{*}[\xi]=\alpha_{*}\left(\rho_{*}[\xi]\right)=\alpha_{*}[\xi]$ if $\rho_{*}[\xi]=[\xi]$.

$(1) \Rightarrow(2)$. Apply (1) to $Y=X$ and the class of the diagonal of $X$ in place of $\alpha$.

Corollary 2.2. If $\rho$ satisfies the conditions of Lemma 2.1, then for any F-variety $Y$ the pull-back homomorphism $\mathrm{CH}(X \times Y) \circ \rho \rightarrow \mathrm{CH}\left(Y_{F(X)}\right)$ is surjective.

Definition 2.3. A smooth complete $F$-variety $X$ is $A$-trivial, if for any field extension $L / F$ with $X(L) \neq \emptyset$, the degree homomorphism deg: $\mathrm{CH}_{0}\left(X_{L}\right) \rightarrow \Lambda$ is an isomorphism.

Remark 2.4. The notion of $A$-triviality depends on $\Lambda$. A variety $A$-trivial for $\Lambda=\mathbb{Z}$ is $A$-trivial for any $\Lambda$. If $\Lambda \neq 0$, any $A$-trivial variety is geometrically irreducible.

Example 2.5. Any projective homogeneous variety $X$ under an action of a semisimple affine algebraic group is $A$-trivial. Indeed, if $X(L) \neq \emptyset$, the variety $X_{L}$ is rational and therefore deg: $\mathrm{CH}_{0}\left(X_{L}\right) \rightarrow \Lambda$ is an isomorphism by Corollary RC.13.

Multiplicity mult $\rho$ of $\rho$ is the element of $\Lambda$ such that the push-forward of $\rho$ with respect to the first projection $X \times X \rightarrow X$ is equal to (mult $\rho) \cdot[X]$.

Lemma 2.6. Assuming that $X$ is A-trivial, $\rho$ satisfies conditions of Lemma 2.1 if and only if mult $\rho=1$. 
Proof. Since $X$ is $A$-trivial, the 0 -cycle classes $\rho_{*}[\xi],[\xi] \in \mathrm{CH}_{0}\left(X_{F(X)}\right)$ coincide if and only if their degrees coincide. It remains to notice that $\operatorname{deg}[\xi]=1$ and $\operatorname{deg} \rho_{*}[\xi]=\operatorname{mult} \rho$.

A trivial example of $\rho$ satisfying the conditions of Lemma 2.1 is given by the class of the diagonal of $X$. Here is one more example:

Example 2.7. If $X$ is a projective homogeneous variety under an action of a semisimple affine algebraic group and $\rho \in \mathrm{CH}^{d}(X \times X)$ is a projector such that the summand $(X, \rho)$ of the Chow motive of $X$ is upper in the sense of [13, Definition 2.10], then mult $\rho=1$ and therefore $\rho$ satisfies conditions of Lemma 2.1 by Lemma 2.6 ( $X$ is $A$-trivial by Example 2.5).

Proposition 2.8. Assume that $\rho$ satisfies conditions of Lemma 2.1 (the assumption is satisfied, for instance, if mult $\rho=1$ and $X$ is A-trivial). Also assume that $\rho$ is a projector. Given an equidimensional $F$-variety $Y$ and an integer $m$ such that for any $i$ and any point $y \in Y$ of codimension $i$ the change of field homomorphism

$$
\rho^{*} \mathrm{CH}^{m-i}(X) \rightarrow \rho^{*} \mathrm{CH}^{m-i}\left(X_{F(y)}\right)
$$

is surjective, the change of field homomorphism

$$
\mathrm{CH}^{m}(Y) \rightarrow \mathrm{CH}^{m}\left(Y_{F(X)}\right)
$$

is also surjective.

Proof. Since $\rho^{*}(x) \times y=(x \times y) \circ \rho$ for any $x \in \mathrm{CH}(X), F$-variety $Y$ and $y \in \mathrm{CH}(Y)$ (where the composition of correspondences is taken in the sense of [3], see also [7, §62]), the external product homomorphism $\mathrm{CH}(X) \otimes \mathrm{CH}(Y) \rightarrow \mathrm{CH}(X \times Y)$ maps $\left(\rho^{*} \mathrm{CH}(X)\right) \otimes$ $\mathrm{CH}(Y)$ to $\mathrm{CH}(X \times Y) \circ \rho$.

Let us check that in our situation the homomorphism

$$
\bigoplus_{i}\left(\rho^{*} \mathrm{CH}^{i}(X)\right) \otimes_{\Lambda} \mathrm{CH}^{m-i}(Y) \rightarrow \mathrm{CH}^{m}(X \times Y) \circ \rho
$$

is surjective.

Checking this, we may assume that $Y$ is integral and proceed by induction on $\operatorname{dim} Y$ using the exact sequence

$$
\bigoplus_{Y^{\prime}} \mathrm{CH}^{m-1}\left(X \times Y^{\prime}\right) \circ \rho \rightarrow \mathrm{CH}^{m}(X \times Y) \circ \rho \rightarrow \rho^{*} \mathrm{CH}^{m}\left(X_{F(Y)}\right),
$$

where the direct sum is taken over all integral subvarieties $Y^{\prime} \subset Y$ of codimension 1. The sequence is exact because the sequence

$$
\bigoplus_{Y^{\prime}} \mathrm{CH}^{m-1}\left(X \times Y^{\prime}\right) \rightarrow \mathrm{CH}^{m}(X \times Y) \rightarrow \mathrm{CH}^{m}\left(X_{F(Y)}\right)
$$

is exact and $\rho$ is a projector.

Now we consider the following commutative diagram

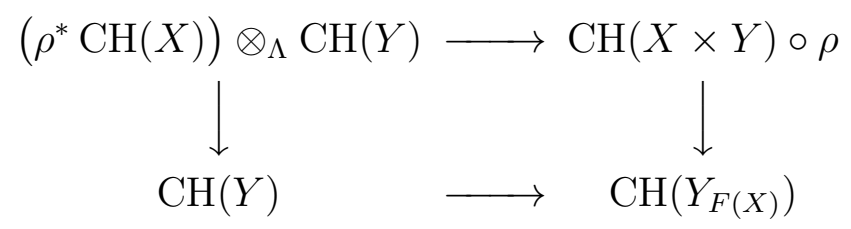


where the left homomorphism is induced by the augmentation map $\mathrm{CH}(X) \rightarrow \Lambda$. The right homomorphism is surjective by Corollary 2.2. As we checked right above, the top homomorphism is surjective in codimension $m$. Therefore the bottom homomorphism is also surjective in codimension $m$.

The following statement is a particular case of $[19$, Theorem $2.11(3 \Rightarrow 1)]$ :

Lemma 2.9. Assume that $X$ is A-trivial and $1 \in \operatorname{deg} \mathrm{CH}_{0}(X)$. Then for any $F$-variety $Y$, the change of field homomorphism $\mathrm{CH}(Y) \rightarrow \mathrm{CH}\left(Y_{F(X)}\right)$ is an isomorphism.

Proof. To prove surjectivity, we note that any $y \in \mathrm{CH}\left(Y_{F(X)}\right)$ is the image of some $\alpha \in$ $\mathrm{CH}(X \times Y)$. If $x \in \mathrm{CH}_{0}(X)$ is an element of degree 1 , then the correspondence $[X] \times x \in$ $\mathrm{CH}^{d}(X \times X)$ satisfies by Lemma 2.6 the conditions of Lemma 2.1. Therefore $\alpha \circ([X] \times x) \in$ $\mathrm{CH}(X \times Y)$ is also mapped to $y \in \mathrm{CH}\left(Y_{F(X)}\right)$. On the other hand, $\alpha \circ([X] \times x)=$ $[X] \times \alpha_{*}(x)$ is mapped to $\alpha_{*}(x)_{F(X)}$ and it follows that $\alpha_{*}(x)$ is an element of $\mathrm{CH}(Y)$ mapped to $y$.

Injectivity follows by specialization (see [9, §20.3] or [27]).

Corollary 2.10. Assume that $X$ is A-trivial. Then for any $l \in \operatorname{deg} \mathrm{CH}_{0}(X) \subset \Lambda$ and any $F$-variety $Y$, the image of $\mathrm{CH}(Y) \rightarrow \mathrm{CH}\left(Y_{F(X)}\right)$ contains $l \mathrm{CH}\left(Y_{F(X)}\right)$.

Proof. It suffices to consider the case $l=\operatorname{deg} x$ for a closed point $x \in X$. Let $L$ be the residue field of $x$. The change of field homomorphism $\mathrm{CH}\left(Y_{L}\right) \rightarrow \mathrm{CH}\left(Y_{L(X)}\right)$ is surjective by Lemma 2.9, and the transfer argument does the job.

\section{Abstract Rost motives}

In this section, the coefficient ring $\Lambda$ is $\mathbb{Z}_{(p)}$ (the ring of integers localized in a fixed prime $p$ ) or $\mathbb{F}_{p}$ (the finite field of $p$ elements).

For any integer $n \geq 1$, an abstract Rost motive of degree $n+1$ with coefficients in $\Lambda$ is a Chow motive $\mathcal{R}$ with coefficients in $\Lambda$ living on a smooth complete geometrically irreducible variety $X$ such that for any field extension $L / F$ with $1 \in \operatorname{deg} \mathrm{CH}_{0}\left(X_{L}\right)$ one has $\mathcal{R}_{L} \simeq \Lambda \oplus \Lambda(b) \oplus \cdots \oplus \Lambda((p-1) b)$, where $b:=\left(p^{n}-1\right) /(p-1)$.

In particular, $\operatorname{dim} X \geq p^{n}-1=(p-1) b$. Pulling-back the projector of $\mathcal{R}$ with respect to the diagonal of $X$, produces a 0 -cycle class of degree $p$ (cf. [13, Lemma 2.21]) showing that $\operatorname{deg} \mathrm{CH}_{0}(X) \supset p \Lambda$. It follows that the the ideal $\operatorname{deg} \mathrm{CH}_{0}(X) \subset \Lambda$ of the coefficient ring $\Lambda$ is equal either to $p \Lambda$ or to $\Lambda$.

The condition $1 \in \operatorname{deg} \mathrm{CH}_{0}\left(X_{L}\right)$ appearing in the definition means the same for $\Lambda=\mathbb{Z}_{(p)}$ as for $\Lambda=\mathbb{F}_{p}$. In $\Lambda$-free terms, it means that the variety $X_{L}$ has a closed point of a prime to $p$ degree.

By the very definition, the multiplicity of the projector of $\mathcal{R}$ (which we call an abstract Rost projector) is equal to 1.

Note that for any field extension $L / F$ such that $1 \in \operatorname{deg} \mathrm{CH}_{0}\left(X_{L}\right)$ we have

$$
\text { End } \mathcal{R}_{L}=\text { End } \Lambda \times \text { End } \Lambda(b) \times \cdots \times \text { End } \Lambda((p-1) b)=\Lambda^{p} .
$$

In particular, End $\mathcal{R}_{L} \rightarrow$ End $\mathcal{R}_{L^{\prime}}$ is an isomorphism for any field extension $L^{\prime} / L$.

We fix an integer $n \geq 1$ and consider only abstract Rost motives of degree $n+1$ below. 
Lemma 3.1. An abstract Rost motive is indecomposable if (and only if) $1 \notin \operatorname{deg} \mathrm{CH}_{0}(X)$. In particular, an abstract Rost motive with coefficients in $\mathbb{Z}_{(p)}$ is indecomposable if and only if the corresponding abstract Rost motive with coefficients in $\mathbb{F}_{p}$ is indecomposable.

Proof. Assuming that $\mathcal{R}=R_{1} \oplus R_{2}$ with $R_{1}, R_{2} \neq 0$, we get $\left(R_{1}\right)_{F(X)},\left(R_{2}\right)_{F(X)} \neq 0$ by the nilpotence principle [32, Proposition 3.1]. It follows by the Krull-Schmidt principle of [5] that $\left(R_{1}\right)_{F(X)}$ is isomorphic to a direct sum of shifts of $m$ copies of $\Lambda$ where $0<m<p$. Pulling back the projector of $R_{1}$ via the diagonal of $X$, we get a 0 -cycle of degree $m \in \Lambda$ (cf. [13, Lemma 2.21]). This contradicts $1 \notin \operatorname{deg} \mathrm{CH}_{0}(X)$.

Lemma 3.2. For any abstract Rost motive $\mathcal{R}$ one has

$$
p \text { End } \mathcal{R}_{F(X)} \subset \operatorname{Im}\left(\text { End } \mathcal{R} \rightarrow \text { End } \mathcal{R}_{F(X)}\right) .
$$

Proof. The statement being vacuous for $\Lambda=\mathbb{F}_{p}$, one may assume that $\Lambda=\mathbb{Z}_{(p)}$ in the proof. We also may assume that $\mathcal{R}$ is indecomposable.

Let $L$ be the residue field of a closed point on $X$ of degree not divisible by $p^{2}$ (but, of course, divisible by $p$ ). Since $X(L) \neq \emptyset$, End $\mathcal{R}_{L} \rightarrow$ End $\mathcal{R}_{L(X)}$ is an isomorphism. For any $\alpha \in$ End $\mathcal{R}_{F(X)}$ the endomorphism $p \alpha$ is in the image of the coinciding with multiplication by $[L: F]$ composition End $\mathcal{R}_{F(X)} \rightarrow$ End $\mathcal{R}_{L(X)} \rightarrow$ End $\mathcal{R}_{F(X)}$ and therefore in the image of the composition End $\mathcal{R}_{L} \rightarrow$ End $\mathcal{R}_{L(X)} \rightarrow$ End $\mathcal{R}_{F(X)}$ which coincides with the composition End $\mathcal{R}_{L} \rightarrow$ End $\mathcal{R} \rightarrow$ End $\mathcal{R}_{F(X)}$.

Lemma 3.3. Any multiplicity 1 endomorphism of an indecomposable abstract Rost motive is an automorphism.

Proof. We take some $\alpha \in$ End $\mathcal{R}$ of multiplicity 1. Since the ring End $\mathcal{R}_{F(X)}$ is the product of $p$ copies of $\Lambda$, the endomorphism $\alpha_{F(X)} \in$ End $\mathcal{R}_{F(X)}$ is given by a $p$-tuple of elements in $\Lambda$. This $p$-tuple starts with 1 (because the starting component of the $p$ tuple is the multiplicity of $\alpha$ ). Actually, every component of the $p$-tuple is congruent to 1 modulo $p$. Indeed, if a component of $\alpha_{F(X)}$ is $\lambda \not \equiv 1$, the $F$-rational (i.e., coming from $F$ ) endomorphism $(\alpha-\lambda \cdot \mathrm{id})_{F(X)}$ considered in $\left(\text { End } \mathcal{R}_{F(X)}\right)_{\Lambda} \otimes \mathbb{F}_{p}$ has a nontrivial and a trivial component. Rasing to $(p-1)$ th power, provides us with a nontrivial $F$ rational idempotent. By the mentioned in the proof of Lemma 3.1 nilpotence principle, this produces a nontrivial idempotent in the ring $(\text { End } \mathcal{R})_{\Lambda} \otimes \mathbb{F}_{p}$, contradicting Lemma 3.1.

So, every component of $\alpha_{F(X)}$ is congruent to 1 modulo $p$ (and this is the end of the proof in the case of $\Lambda=\mathbb{F}_{p}$ ). In particular, $\alpha_{F(X)}$ is invertible. By Lemma 3.2, the inverse of $\alpha_{F(X)}$ is rational (because each component of the inverse is also congruent to 1 modulo $p$ ). Therefore we may assume that $\alpha_{F(X)}=1$. In this case, $\alpha-1$ is nilpotent by nilpotence principle applied one more time, and it follows that $\alpha$ itself is invertible.

It turns out that $\mathcal{R}$ is determined by the class of $X$ with respect to the following equivalence relation: $X \sim X^{\prime}$ if there exist multiplicity 1 correspondences $X \rightsquigarrow X^{\prime}$ and $X^{\prime} \rightsquigarrow X$. (In slightly different terms, $X \sim X^{\prime}$ means that for any field extension $L / F$ one has $1 \in \operatorname{deg} \mathrm{CH}_{0}\left(X_{L}\right)$ if and only if $1 \in \operatorname{deg} \mathrm{CH}_{0}\left(X_{L}^{\prime}\right)$. Note that the equivalence relations for $\Lambda=\mathbb{Z}_{(p)}$ and $\Lambda=\mathbb{F}_{p}$ coincide.) More precisely, we have 
Proposition 3.4. Abstract Rost motives $\mathcal{R}$ and $\mathcal{R}^{\prime}$ living on varieties $X$ and $X^{\prime}$ are isomorphic if and only if the varieties are equivalent. If an indecomposable abstract Rost motive lives on one of two equivalent varieties, then it also lives on the other one.

Proof. Mutually inverse isomorphisms between $\mathcal{R}$ and $\mathcal{R}^{\prime}$ living on varieties $X$ and $X^{\prime}$ are given by some correspondences $f: X \rightsquigarrow X^{\prime}$ and $g: X^{\prime} \rightsquigarrow X$. Since $\mathcal{R}=(X, g \circ f)$, we have $\operatorname{mult}(g \circ f)=1$. As $\operatorname{mult}(g \circ f)=\operatorname{mult}(g) \cdot \operatorname{mult}(f)$, the correspondences $f$ and $g$ have prime to $p$ multiplicities showing that $X \sim X^{\prime}$.

Now given an indecomposable $\mathcal{R}$ living on $X$ and given some $X^{\prime}$ equivalent to $X$, we show that $\mathcal{R}$ is a direct summand of the motive $M\left(X^{\prime}\right)$ of $X^{\prime}$. The equivalence $X \sim X^{\prime}$ provides us with multiplicity 1 correspondences $f: X \rightsquigarrow X^{\prime}$ and $g: X^{\prime} \rightsquigarrow X$. The composition $g \circ f$ considered on $\mathcal{R}$ is a multiplicity 1 endomorphism of $\mathcal{R}$. This endomorphism is an automorphism by Lemma 3.3 .

Finally, if we are given some $\mathcal{R}$ and $\mathcal{R}^{\prime}$ living on some equivalent $X$ and $X^{\prime}$, and we want to show that $\mathcal{R} \simeq \mathcal{R}^{\prime}$, then we may assume that $\mathcal{R}$ and $\mathcal{R}^{\prime}$ are indecomposable and consider morphisms $\mathcal{R} \rightarrow \mathcal{R}^{\prime}$ and $\mathcal{R}^{\prime} \rightarrow \mathcal{R}$ given by multiplicity 1 correspondences $f: X \rightsquigarrow X^{\prime}$ and $g: X^{\prime} \rightsquigarrow X$. Repeating the above argument, we show that $\mathcal{R}$ is a direct summand of $\mathcal{R}^{\prime}$. Therefore $\mathcal{R} \simeq \mathcal{R}^{\prime}$ by indecomposability of $\mathcal{R}^{\prime}$.

Corollary 3.5. Abstract Rost motives with coefficients in $\mathbb{Z}_{(p)}$, becoming isomorphic after the change of coefficients $\mathbb{Z}_{(p)} \rightarrow \mathbb{F}_{p}$, are isomorphic.

We recall that canonical $p$-dimension $\operatorname{cd}_{p} X$ of a smooth complete irreducible variety $X$ is the least dimension of a closed subvariety $Y \subset X$ possessing a multiplicity 1 correspondence $X \rightsquigarrow Y$, cf. [16]. One always has $\operatorname{cd}_{p} X \leq \operatorname{dim} X$, and $X$ is called $p$-incompressible in the case of equality. Canonical $p$-dimensions of equivalent varieties coincide:

Lemma 3.6. If $X \sim X^{\prime}$, then $\operatorname{cd}_{p} X=\operatorname{cd}_{p} X^{\prime}$.

Proof. Assuming that $X \sim X^{\prime}$, it suffices to show that $\operatorname{cd}_{p} X \leq \operatorname{cd}_{p} X^{\prime}$. Let $Y^{\prime}$ be a closed irreducible subvariety of $X^{\prime}$ with a multiplicity 1 correspondence $X^{\prime} \rightsquigarrow Y^{\prime}$ and with $\operatorname{dim} Y^{\prime}=\operatorname{cd}_{p} X^{\prime}$. Then there exists a prime correspondence $X^{\prime} \rightsquigarrow Y^{\prime}$ of prime to $p$ multiplicity. Such a correspondence is given by an irreducible closed subvariety $Z^{\prime}$ in $X^{\prime} \times Y^{\prime}$ such that the projection $Z^{\prime} \rightarrow X^{\prime}$ is surjective and the field extension $F\left(X^{\prime}\right) \hookrightarrow F\left(Z^{\prime}\right)$ is of finite prime to $p$ degree. By minimality of $Y^{\prime}$, the projection $Z^{\prime} \rightarrow Y^{\prime}$ is also surjective.

The variety $X^{\prime}$ having an $F\left(Y^{\prime}\right)$-point, the variety $X_{F\left(Y^{\prime}\right)}$ has a 0-cycle of degree 1 . Consequently, there exists a closed subvariety $Z \subset Y^{\prime} \times X$ surjective over $Y^{\prime}$ with $F\left(Y^{\prime}\right) \hookrightarrow$ $F(Z)$ of finite prime to $p$ degree. Let $Y \subset X$ be the image of the projection $Z \rightarrow X$.

We have obtained a diagram of fields, shown below on the left, in which the vertical embeddings are of finite prime to $p$ degrees. By [16, Lemma 3.1], it can be completed to a commutative diagram of fields, shown on the right, in which the vertical embeddings 
are still of finite prime to $p$ degrees:
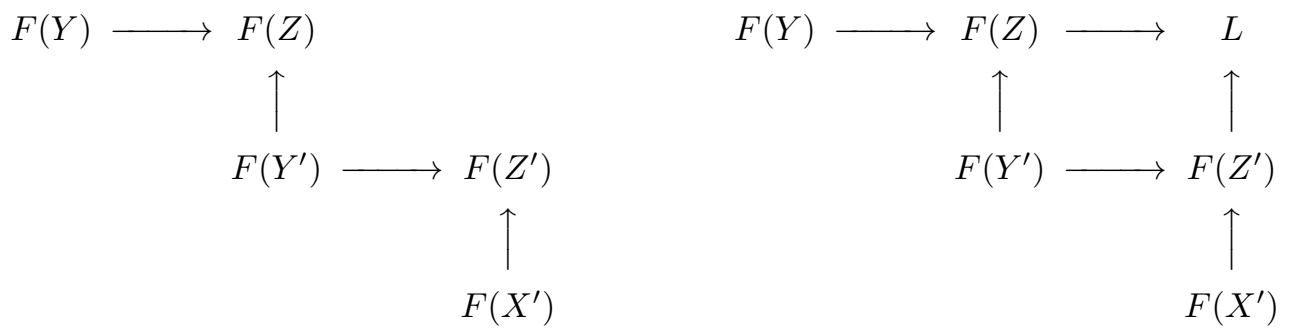

Taking a model $U$ for $L$ and considering the class of the closure of the image of the induced rational map $U \rightarrow X^{\prime} \times Y$, we get a prime correspondence $X^{\prime} \rightsquigarrow Y$ of a prime to $p$ multiplicity, showing that there exists a multiplicity 1 correspondence $X^{\prime} \rightsquigarrow Y$. Composing it with a multiplicity 1 correspondence $X \rightsquigarrow X^{\prime}$ (the composition is defined because $X^{\prime}$ is smooth complete), we get a multiplicity 1 correspondence $X \rightsquigarrow Y$ showing that $\operatorname{cd}_{p} X \leq \operatorname{dim} Y \leq \operatorname{dim} Z=\operatorname{dim} Y^{\prime}=\operatorname{cd}_{p} X^{\prime}$.

Remark 3.7. Lemma 3.6 is easier to prove out of the (equivalent) definition of canonical $p$-dimension of a smooth complete variety $X$ as the essential p-dimension of the class of fields $L / F$ with $X(L) \neq \emptyset$, given in [21, §1.6]. Indeed, enlarging the above class of fields to the class of fields $L / F$ with $1 \in \operatorname{deg} \mathrm{CH}_{0}\left(X_{L}\right)$ keeps its essential $p$-dimension. And, as we already mentioned, such enlarged classes of fields given by equivalent varieties coincide.

Lemma 3.8. If an indecomposable abstract Rost motive (of degree $n+1$ ) lives on a variety $X$, then $\operatorname{cd}_{p} X \geq p^{n}-1$.

Proof. For a closed subvariety $Y \subset X$ with a multiplicity 1 correspondence $X \rightsquigarrow Y$, we consider the endomorphism $\alpha \in$ End $\mathcal{R}$ given by the composition of correspondences $X \rightsquigarrow$ $Y \hookrightarrow X$. (More explicitly, $\alpha$ is the composition $X \rightsquigarrow X \rightsquigarrow Y \hookrightarrow X \rightsquigarrow X$ with $X \rightsquigarrow X$ being the projector of $R$.) Since mult $\alpha=1, \alpha$ is invertible by Lemma 3.3. On the other hand, the very last component of $\alpha_{F(X)}$ can be nonzero only if $\operatorname{dim} Y \geq p^{n}-1$. Indeed, this last component is given by the action of $\alpha_{F(X)}^{*}$ on $\mathrm{CH}^{p^{n}-1}\left(\mathcal{R}_{F(X)}\right) \subset \mathrm{CH}^{p^{n}-1}\left(X_{F(X)}\right)$, but since $\alpha$ lies in the image of the push-forward $\mathrm{CH}(X \times Y) \rightarrow \mathrm{CH}(X \times X)$, the action on the whole group $\mathrm{CH}^{p^{n}-1}\left(X_{F(X)}\right)$ is trivial if $\operatorname{dim} Y<p^{n}-1$.

Corollary 3.9. If an indecomposable abstract Rost motive lives on a variety $X$ of dimension $p^{n}-1$, then $X$ is $p$-incompressible.

The following Lemma is inspired by [26, Lemma 9.3]:

Lemma 3.10. If $X$ is $p$-incompressible, then for any $i>0$ and any $\alpha \in \mathrm{CH}^{i}(X)$ and $\beta \in$ $\mathrm{CH}_{i}\left(X_{F(X)}\right)$, the degree of the 0 -cycle class given by the product $\alpha_{F(X)} \cdot \beta \in \mathrm{CH}_{0}\left(X_{F(X)}\right)$ is divisible by $p$.

Proof. If $\operatorname{deg}\left(\alpha_{F(X)} \cdot \beta\right)$ is not divisible by $p$ for some $\alpha \in \mathrm{CH}^{i}(X)$ and $\beta \in \mathrm{CH}_{i}\left(X_{F(X)}\right)$ with positive $i$, we can find a closed irreducible subvariety $Y \subset X$ of codimension $i$ with $\operatorname{deg}\left([Y]_{F(X)} \cdot \beta\right)$ not divisible by $p$. Since the product $[Y]_{F(X)} \cdot \beta$ is represented by a 0 cycle class on $Y_{F(X)}$, there exists a multiplicity 1 correspondence $X \rightsquigarrow Y$ showing that $\operatorname{cd}_{p} X \leq \operatorname{dim} X-i<\operatorname{dim} X$ contradicting the assumption that $X$ is $p$-incompressible. 
Corollary 3.11 (cf. [26, Lemma 9.3]). If an abstract Rost motive lives on a variety $X$ of dimension $p^{n}-1$ and such that $1 \notin \operatorname{deg} \mathrm{CH}_{0}(X)$, then for any $i>0$ and any $\alpha \in \mathrm{CH}^{i}(X)$ and $\beta \in \mathrm{CH}_{i}\left(X_{F(X)}\right)$, the degree of the product $\alpha_{F(X)} \cdot \beta \in \mathrm{CH}_{0}\left(X_{F(X)}\right)$ is divisible by $p$.

\section{Generic splitting Varieties}

In this section $\Lambda$ is $\mathbb{Z}_{(p)}$ or $\mathbb{F}_{p}$ and the base field $F$ is of characteristic $\neq p$ if not specified otherwise.

For $n \geq 1$, an element $s \in H^{n+1}\left(F, \mu_{p}^{\otimes n}\right)$ is a symbol, if it is equal to the cup product of an element of $H^{1}(F, \mathbb{Z} / p \mathbb{Z})$ and $n$ elements of $H^{1}\left(F, \mu_{p}\right)$. A smooth complete geometrically irreducible $F$-variety $X$ is a p-generic splitting variety of a symbol $s$, if for any field extension $L / F$ one has $s_{L}=0$ if and only if $1 \in \operatorname{deg} \mathrm{CH}_{0}\left(X_{L}\right)$ (it is a generic splitting variety of $s$, if $\left.s_{L}=0 \Leftrightarrow X(L) \neq \emptyset\right)$.

Clearly, given a symbol $s$ and a p-generic splitting variety $X$ of $s$, a smooth complete geometrically irreducible variety $X^{\prime}$ is also a $p$-generic splitting variety of $s$ if and only if $X \sim X^{\prime}$

A symbol $s^{\prime}$ is similar to $s$, if $s^{\prime}=a s$ for a nonzero $a \in \mathbb{Z} / p \mathbb{Z}$. Similar symbols vanish over precisely the same fields so that $p$-generic splitting varieties of similar symbols are equivalent.

According to [28], in characteristic 0 , for any symbol $s$, there exists a $p$-generic splitting variety of dimension $p^{n}-1$. The construction of such varieties is recalled in Section $5 \mathrm{~d}$.

An abstract Rost motive $\mathcal{R}=\mathcal{R}_{s}$ living on a $p$-generic splitting variety of a symbol $s \in H^{n+1}\left(F, \mu_{p}^{\otimes n}\right)$ is called a Rost motive of the symbol.

Theorem 4.1. Assume that char $F=0$. For any symbol $s \in H^{n+1}\left(F, \mu_{p}^{\otimes n}\right)$, a Rost motive $\mathcal{R}_{s}$ of $s$ exists. Moreover, the isomorphism class of $\mathcal{R}_{s}$ determines and is determined by the similarity class of $s$.

Proof. The existence statement is proved in [33] and [28]. By Proposition 3.4, the isomorphism class of $\mathcal{R}_{s}$ is determined by the similarity class of $s$. Finally, if $\mathcal{R}_{s^{\prime}} \simeq \mathcal{R}_{s}$ for a symbol $s^{\prime}$, the symbols $s$ and $s^{\prime}$ vanish over precisely the same field extensions of $F$ and therefore are similar by [20, Theorem 2.1].

Remark 4.2. In characteristic 0 one may show using Theorem RM.10 that for any Rost motive $\mathcal{R}$ of a nonzero symbol living on a variety $X$, the homomorphism of $\mathbb{Z}_{(p)}$-algebras

$$
\text { End } \mathcal{R} \rightarrow \text { End } \mathcal{R}_{F(X)}=\mathbb{Z}_{(p)}^{p}
$$

is injective and has as image the unital $\mathbb{Z}_{(p)}$-subalgebra of $\mathbb{Z}_{(p)}^{p}$ generated by $p \mathbb{Z}_{(p)}^{p}$. This explains Lemmas 3.2 and 3.3 .

Keeping the characteristic 0 assumption, it follows by Proposition 3.4 that any $p$-generic splitting variety of $s$ admits a Rost motive and the isomorphism class of a Rost motive on such a variety only depends on $s$. It follows also that $p^{n}-1$ is the least dimension of a $p$-generic splitting variety of a symbol. The $p$-generic splitting varieties of dimension $p^{n}-1$ are called norm varieties. 
Theorem 4.3 (Version for $\Lambda=\mathbb{Z}_{(p)}$ and $\Lambda=\mathbb{F}_{p}$ ). Let $F$ be a field of characteristic 0 . Given an A-trivial p-generic splitting variety $X$ of a symbol $s \in H^{n+1}\left(F, \mu_{p}^{\otimes n}\right)$, the change of field homomorphism $\mathrm{CH}(Y) \rightarrow \mathrm{CH}\left(Y_{F(X)}\right)$ is surjective in codimensions < $\left(p^{n}-1\right) /(p-1)$ for any equidimensional variety $Y$. It is also surjective in codimension $=\left(p^{n}-1\right) /(p-1)$ for a given $Y$ provided that $s_{F(\zeta)} \neq 0$ for each generic point $\zeta \in Y$.

Proof. If $s=0$, then $1 \in \operatorname{deg} \mathrm{CH}_{0}(X)$ and the statement of Theorem 4.3 is a particular case of Lemma 2.9. Below in the proof we are assuming that $s \neq 0$.

Let $\rho$ be a projector on $X$ giving the Rost motive. By Proposition 2.8, to prove the first statement of Theorem 4.3 , it suffices to check that for any field extension $L / F$ the change of field homomorphism

$$
\rho^{*} \mathrm{CH}(X) \rightarrow \rho^{*} \mathrm{CH}\left(X_{L}\right)
$$

is surjective in codimension $<m:=\left(p^{n}-1\right) /(p-1)$. This condition is satisfied by Theorem RM.10.

To prove the second statement of Theorem 4.3, it suffices to additionally check that for any generic point $\zeta \in Y$ the change of field homomorphism

$$
\rho^{*} \mathrm{CH}^{m}(X) \rightarrow \rho^{*} \mathrm{CH}^{m}\left(X_{F(\zeta)}\right)
$$

is surjective. Since $s_{F(\zeta)} \neq 0$, this condition is satisfied by Theorem RM.10 as well.

Our main example of $X$ for which Theorem 4.3 can be applied is given by the standard norm variety of a symbol in $H^{n+1}\left(F, \mu^{\otimes n}\right)$, constructed in Section 5d. Such a variety is $A$-trivial by Theorem 5.8.

The standard norm variety $X$ of a nonzero symbol also provides an example showing that the boundary $b:=\left(p^{n}-1\right) /(p-1)$ of the first (and main) statement of Theorem 4.3 is sharp. Indeed, the element $H \in \mathrm{CH}^{b}\left(X_{F(X)}\right)$ considered in Section $\mathbf{S q}$, does not come from $F$.

A construction similar to [31, Proof of Theorem 3.4], proves

Corollary 4.4. For any field $F$ of characteristic 0 , any prime $p$ and any integer $n \geq 1$, there exists a field extension $F^{\prime} / F$ such that $H^{n+1}\left(F^{\prime}, \mu_{p}^{\otimes n}\right)=0$ and $\mathrm{CH}(Y) \rightarrow \mathrm{CH}\left(Y_{F^{\prime}}\right)$ is surjective in codimensions $<\left(p^{n}-1\right) /(p-1)$ for any equidimensional $F$-variety $Y$.

As indicated in [31, Remark after Theorem 3.4], Corollary 4.4 shows that "modulo $p$ and degree $>n$ cohomological invariants of equidimensional algebraic varieties could not affect rationality of cycles of codimension $<\left(p^{n}-1\right) /(p-1)$ ".

Theorem 4.5 (Version for $\Lambda=\mathbb{Z}$ ). We have $\Lambda=\mathbb{Z}$ in this statement. Let $F$ be a field of characteristic 0. Given an A-trivial p-generic splitting variety $X$ of a symbol $s \in H^{n+1}\left(F, \mu_{p}^{\otimes n}\right)$ such that $p \in \operatorname{deg} \mathrm{CH}_{0}(X)$, the homomorphism $\mathrm{CH}(Y) \rightarrow \mathrm{CH}\left(Y_{F(X)}\right)$ is surjective in codimensions $<\left(p^{n}-1\right) /(p-1)$ for any equidimensional variety $Y$. It is also surjective in codimension $=\left(p^{n}-1\right) /(p-1)$ for a given $Y$ provided that $s_{F(\zeta)} \neq 0$ for each generic point $\zeta \in Y$.

Proof. By Theorem 4.3, $\mathrm{CH}(Y) \rightarrow \mathrm{CH}\left(Y_{F(X)}\right)$ is surjective modulo $p$ in the codimensions considered. Since $p \in \operatorname{deg} \mathrm{CH}_{0}(X)$, the image of $\mathrm{CH}(Y) \rightarrow \mathrm{CH}\left(Y_{F(X)}\right)$ contains $p \mathrm{CH}\left(Y_{F(X)}\right)$ by Corollary 2.10. 
Example 4.6. For $p=2$, let $a_{0}, \ldots, a_{n} \in F^{\times}, s$ the symbol $s:=\left(a_{0}\right) \cup \cdots \cup\left(a_{n}\right)$, and $X$ the norm quadric $\left\langle-a_{0}\right\rangle \perp\left\langle\left\langle a_{1}, \ldots, a_{n}\right\rangle\right\rangle=0$. Then $X$ is an $A$-trivial (see Example 2.5) norm variety of $s$ with $2 \in \operatorname{deg} \mathrm{CH}_{0}(X)$ so that Theorem 4.5 applies. The result obtained has been originally established by A. Vishik in [31, Corollary 3.3]. Using [12] in place of Appendix RM, the characteristic 0 assumption can be replaced by characteristic $\neq 2$ assumption in this statement.

Example 4.7. Let char $F=0, p=3$, and let $X$ be any (among 15) nontrivial projective homogeneous $F$-variety under an action of a given absolutely simple affine algebraic group of type $F_{4}$ over $F$. Then the conclusion of Theorem 4.3 holds for $X$ with $n=2$. Indeed, $X$ is $A$-trivial (for any coefficient ring) by Example 2.5. The modulo 3 portion of the Rost invariant (see [18]) provides us with an element $s \in H^{3}\left(F, \mu_{3}^{\otimes 2}\right)$. This element is a symbol by [29, p. 303] (see also [10, p. 21]). Moreover, $X$ is a 3-generic splitting variety of $s$ (see [10, $\S 15.5]$ ), so that Theorem 4.3 applies. The result obtained is an enhancement (in several respects) of [34, Case $p=3$ of Corollary 1.4)].

If $F$ has no finite extensions of degree prime to 3 , we have $3 \in \operatorname{deg} \mathrm{CH}_{0}(X)$, and Theorem 4.5 applies.

\section{A-triviality of STANDARD NORM VARIETIES}

5a. Retract rational varieties. A variety $X$ over $F$ is called retract rational if there exist rational morphisms $\alpha: X \rightarrow \mathbb{P}^{n}$ and $\beta: \mathbb{P}^{n} \rightarrow X$ for some $n$ such that the composition $\beta \circ \alpha$ is defined and is equal to the identity of $X$.

The following proposition is due to D. Saltman.

Proposition 5.1. Let $A$ be a central simple algebra of prime degree. Then the variety of the algebraic group $\mathbf{S L}_{1}(A)$ is retract rational.

Proof. As $\operatorname{deg}(A)$ is prime, the group $S K_{1}\left(A_{K}\right)$ is trivial for every field extension $K / F$ [6, $\S 23$, Corollary 4]. Taking $K=F(G)$, we can write the generic point $\xi \in \mathbf{S L}_{1}(A)(K)$ as product of commutators

$$
\xi=\left[f_{1}, f_{1}^{\prime}\right] \cdot\left[f_{2}, f_{2}^{\prime}\right] \cdot \ldots \cdot\left[f_{n}, f_{n}^{\prime}\right]
$$

in $\left(A_{K}\right)^{\times}$, where $f_{i}, g_{i} \in\left(A_{K}\right)^{\times}$. The $2 n$-tuple of functions $\left(f_{1}, f_{1}^{\prime}, \ldots, f_{n}, f_{n}^{\prime}\right)$ can be viewed as a rational morphism $\alpha$ from $G$ to the affine space $\mathbb{A}\left(A^{2 n}\right)$ of the direct sum of $2 n$ copies of the vector space of the algebra $A$. Define the rational morphism $\beta: \mathbb{A}\left(A^{2 n}\right)-\rightarrow G$ by

$$
\beta\left(a_{1}, a_{1}^{\prime}, \ldots, a_{n}, a_{n}^{\prime}\right)=\left[a_{1}, a_{1}^{\prime}\right] \cdot \ldots \cdot\left[a_{n}, a_{n}^{\prime}\right] .
$$

By construction, the composition $\beta \circ \alpha$ is defined and is equal to the identity of $G$.

5b. A-trivial varieties revisited. Recall that a smooth complete variety $X$ over $F$ is called $A$-trivial if for every field extension $K / F$ such that $X(K) \neq \emptyset$, the degree homomorphism $\mathrm{CH}_{0}\left(X_{K}\right) \rightarrow \Lambda$ is an isomorphism.

Example 5.2. A retract rational smooth complete variety $X$ is $A$-trivial. Indeed, it suffices to prove this for $\Lambda=\mathbb{Z}$. Let $\alpha: X \rightarrow \mathbb{P}^{n}$ and $\beta: \mathbb{P}^{n} \rightarrow X$ be rational 
morphisms such that the composition $\beta \circ \alpha$ is defined and is equal to the identity of $X$, then the composition (see Appendix RC)

$$
\mathrm{CH}_{0}\left(X_{K}\right) \stackrel{\alpha_{*}}{\rightarrow} \mathrm{CH}_{0}\left(\mathbb{P}_{K}^{n}\right) \stackrel{\beta_{*}}{\rightarrow} \mathrm{CH}_{0}\left(X_{K}\right)
$$

is the identity for any field extension $K / F$. As $\mathrm{CH}_{0}\left(\mathbb{P}_{K}^{n}\right)=\mathbb{Z}, \alpha_{*}$ is an isomorphism that is equal to the degree map.

Proposition 5.3. A smooth complete variety $X$ over $F$ is A-trivial if and only if for every field extension $K / F$ and every two points $x, x^{\prime} \in X(K)$, we have $[x]=\left[x^{\prime}\right]$ in $\mathrm{CH}_{0}\left(X_{K}\right)$.

Proof. $\Rightarrow:$ As $\operatorname{deg}[x]=1=\operatorname{deg}\left[x^{\prime}\right]$, we have $[x]=\left[x^{\prime}\right]$ in $\mathrm{CH}_{0}\left(X_{K}\right)$.

$\Leftarrow$ : Let $K / F$ be a field extension such that $X(K) \neq \emptyset$. Let $x \in X_{K}$ be a rational point and $y \in X_{K}$ a closed point of degree $m$. It suffices to show that $[y]=m[x]$ in $\mathrm{CH}_{0}\left(X_{K}\right)$. Let $L=K(y)$ and $y^{\prime}$ a rational point of $X_{L}$ over $y$. By assumption $\left[y^{\prime}\right]=\left[x_{L}\right]$ in $\mathrm{CH}_{0}\left(X_{L}\right)$. Applying the push-forward homomorphism $\mathrm{CH}_{0}\left(X_{L}\right) \rightarrow \mathrm{CH}_{0}\left(X_{K}\right)$, we get $[y]=m[x]$.

Proposition 5.4. (1) If $X$ is an A-trivial variety over $F$, then so is $X_{K}$ for any field extension $K / F$.

(2) If $X$ and $X^{\prime}$ are $A$-trivial varieties over $F$, then so is $X \times X^{\prime}$.

(3) Let $E / F$ be a separable field extension and $Y$ a variety over $E$. If $Y$ is A-trivial, then so is the Weil transfer $R_{E / F}(Y)$.

Proof. (1) is trivial.

(2) Let $K / F$ be a field extension and $y_{1}, y_{2} \in\left(X \times X^{\prime}\right)(K)$. We have $y_{1}=\left(x_{1}, x_{1}^{\prime}\right)$ and $y_{2}=\left(x_{2}, x_{2}^{\prime}\right)$ for $x_{1}, x_{2} \in X(K), x_{1}^{\prime}, x_{2}^{\prime} \in X^{\prime}(K)$. As $X$ and $X^{\prime}$ are $A$-trivial, we have $\left[x_{1}\right]=\left[x_{2}\right]$ in $\mathrm{CH}_{0}\left(X_{K}\right),\left[x_{1}^{\prime}\right]=\left[x_{2}^{\prime}\right]$ in $\mathrm{CH}_{0}\left(X_{K}^{\prime}\right)$ and hence

$$
\left[y_{1}\right]=\left[x_{1}\right] \times\left[x_{1}^{\prime}\right]=\left[x_{2}\right] \times\left[x_{2}^{\prime}\right]=\left[y_{2}\right]
$$

in $\mathrm{CH}_{0}\left(X \times X^{\prime}\right)_{K}$.

(3) Let $K / F$ be a field extension and write $E \otimes_{F} K \simeq E_{1} \times \cdots \times E_{s}$, where $E_{i}$ are field extensions of $K$. We have $R_{E / F}(Y)_{K}=R_{E \otimes_{F} K / K}\left(Y_{E \otimes_{F} K}\right)=\prod R_{E_{i} / K}\left(Y_{E_{i}}\right)$. Write two points $y, y^{\prime} \in R_{E / F}(Y)(K)=Y\left(E \otimes_{F} K\right)=\prod Y\left(E_{i}\right)$ in the form $y=\left(y_{1}, \ldots, y_{s}\right)$ and $y^{\prime}=\left(y_{1}^{\prime}, \ldots, y_{s}^{\prime}\right)$, where $y_{i}, y_{i}^{\prime} \in Y\left(E_{i}\right)=R_{E_{i} / K}\left(Y_{E_{i}}\right)(K)$. By assumption, $\left[y_{i}\right]=\left[y_{i}^{\prime}\right]$ in $\mathrm{CH}_{0}\left(Y_{E_{i}}\right)$ for all $i$. Applying the canonical maps

$$
\mathrm{CH}_{0}\left(Y_{E_{i}}\right) \rightarrow \mathrm{CH}_{0}\left(R_{E_{i} / K}\left(Y_{E_{i}}\right)\right)
$$

(see [15]) we get $\left[y_{i}\right]=\left[y_{i}^{\prime}\right]$ in $\mathrm{CH}_{0}\left(R_{E_{i} / K}\left(Y_{E_{i}}\right)\right)$ for all $i$. It follows that $[y]=\left[y^{\prime}\right]$ in $\mathrm{CH}_{0}\left(R_{E / F}(Y)_{K}\right)$.

5c. Symmetric powers. Let $p$ be an integer and let $Y$ be a quasi-projective variety over $F$. Write $S^{p}(Y)$ for the symmetric $p$ th power of $Y$, i.e., the factor variety of $Y^{p}$ by the natural action of the symmetric group $S_{p}$. A $K$-point of $S^{p} Y$ is an effective 0 -cycle on $Y_{K}$ of degree $p$.

Write $\widetilde{Y}^{p}$ for $Y^{p}$ with all the diagonals removed and write $\widetilde{S}^{p}(Y)$ for the factor variety $\widetilde{Y}^{p} / S_{p}$. If $Y$ is smooth, then so is $\widetilde{S}^{p}(Y)$. Every $F$-point $z$ of $\widetilde{S}^{p}(Y)$ gives rise to an effective 0-cycle $y_{1}+y_{2}+\cdots+y_{k}$ on $Y$ of degree $p$ with distinct closed points $y_{1}, y_{2}, \ldots, y_{k}$ on $Y$. We will write $F\{z\}$ for the $F$-algebra $F\left(y_{1}\right) \times F\left(y_{2}\right) \times \cdots \times F\left(y_{k}\right)$ of dimension $p$. 
Consider the natural morphism $g: Y \times S^{p-1}(Y) \rightarrow S^{p}(Y)$. By [28, §2], the sheaf $\mathcal{L}=\left.g_{*}\left(\mathcal{O}_{Y \times S^{p-1}(Y)}\right)\right|_{\widetilde{S}^{p}(Y)}$ is a locally free $\mathcal{O}_{\widetilde{S}^{p}(Y)}$-algebra of rank $p$. The sheaf $\mathcal{L}$ determines a rank $p$ vector bundle $J$ over $\widetilde{S}^{p}(Y)$ such that the fiber of $J$ over a point $z$ in $\widetilde{S}^{p}(Y)$ is the vector space of the algebra $F\{z\}$.

Consider the pull-back diagram

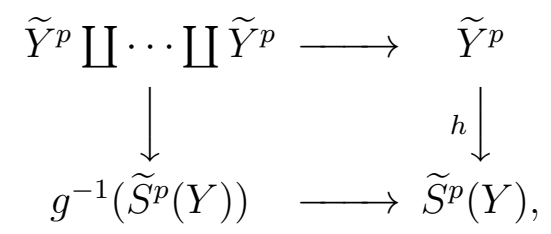

where the left top corner is disjoint union of $p$ copies of $\widetilde{Y}^{p}$, the upper map is the identity on each copy and the left vertical morphism on the $i$ th copy takes a point $\left(y_{1}, \ldots, y_{p}\right)$ to $\left(y_{i}, y_{1}+\cdots+\widehat{y}_{i}+\cdots+y_{p}\right)$. It follows that the sheaf $h^{*}(\mathcal{L})$ of modules on $\widetilde{Y}^{p}$ is free. Therefore, the vector bundle $h^{*}(J)$ on $\widetilde{Y}^{p}$ is trivial.

Let $E$ be an étale $F$-algebra of dimension $p$. Consider the natural morphism $f$ : $R_{E / F}\left(Y_{E}\right) \rightarrow S^{p}(Y)$ (see [11, pp. 267-268]). The map $f$ takes a point $y$ in $R_{E / F}\left(Y_{E}\right)(F)=$ $Y(E)$ to the 0-cycle $y_{1}+\cdots+y_{p}$, where $y_{i}$ are the images of $y$ under all the embeddings of $E$ into $F_{\text {sep }}$. If $E$ is split, $f$ is the natural morphism $Y^{p} \rightarrow S^{p}(Y)$. In general, $f$ can be obtained via the twist of this morphism by the $S_{p}$-torsor corresponding to the étale algebra $E$. Moreover, the étale group scheme $G=\operatorname{Aut}(E / F)$ (the twisted form of the symmetric group $S_{p}$ by the same $S_{p}$-torsor) acts naturally on $R_{E / F}\left(Y_{E}\right)$ and $f$ identifies $S^{p}(Y)$ with the factor variety of $R_{E / F}\left(Y_{E}\right)$ by $G$.

Write $\widetilde{R}_{E / F}\left(Y_{E}\right)$ for the preimage $f^{-1}\left(\widetilde{S}^{p}(Y)\right)$ and $\tilde{f}$ for the morphism $\widetilde{R}_{E / F}\left(Y_{E}\right) \rightarrow$ $\widetilde{S}^{p}(Y)$.

Lemma 5.5. If $z$ is a rational point of $\widetilde{S}^{p}(Y)$ and $E=F\{z\}$, then $z$ lifts to a rational point in $\widetilde{R}_{E / F}\left(Y_{E}\right)$.

Proof. We may assume that $E$ is a field. Over $F_{\text {sep }}, z$ is the cycle $y_{1}+\cdots+y_{p}$, where the distinct points $y \in Y\left(F_{\text {sep }}\right)$ are permuted transitively by the Galois group of $F_{\text {sep }} / F$. Choose a point $y \in Y(E)=\widetilde{R}_{E / F}\left(Y_{E}\right)$ and an embedding of $E$ into $F_{\text {sep }}$ such that the map $Y(E) \rightarrow Y\left(F_{\text {sep }}\right)$ takes $y$ to $y_{1}$. Then $\tilde{f}(y)=y_{1}+\cdots+y_{p}=z$.

Twisting the diagram above by the $S_{p}$-torsor $E$, we see that the pull-back $\tilde{f}^{*}(J)$ is a trivial vector bundle over $\widetilde{R}_{E / F}\left(Y_{E}\right)$ with the fiber $E$, i.e., we have the following fiber product diagram:

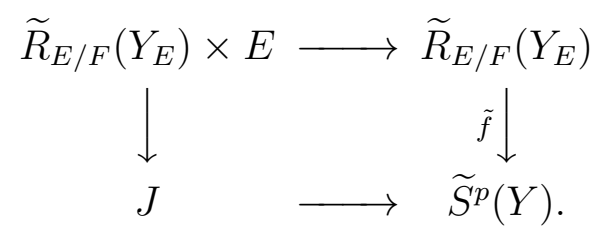

5d. A-triviality of standard norm varieties. We need char $F=0$ here. We recall the construction of certain norm varieties given in [28, §2].

Let $p$ be a prime integer, $Y$ a variety over $F$ and $a \in F^{\times}$. Recall that we have a vector bundle $J$ of rank $p$ over $\widetilde{S}^{p}(Y)$. Write $V(Y, a)$ for the hypersurface in $J$ defined in the 
fiber over every point $z$ of $\widetilde{S}^{p}(Y)$ by the equation $N=a$, where $N$ is the norm map for the algebra $F\{z\}$. If $Y$ is smooth geometrically irreducible variety, then so is $V(Y, a)$ and $\operatorname{dim} V(Y, a)=p(\operatorname{dim} Y+1)-1$ by [28, Lemma 2.1].

Let $E$ be an étale $F$-algebra of dimension $p$ and let $E^{a}$ be the hypersurface in the affine space $\mathbb{A}(E)$ given by the equation $N_{E / F}(x)=a$. We have the following fiber product diagram

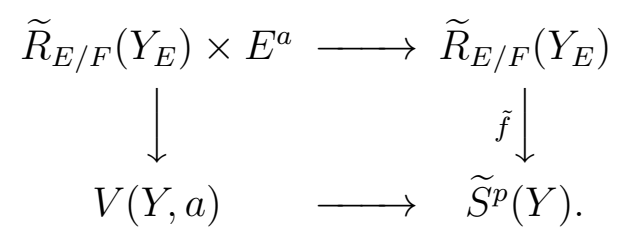

The étale group scheme $G=\operatorname{Aut}(E / F)$ acts naturally on the varieties in the top row and the vertical morphisms are $G$-torsors.

Let $L$ be a cyclic étale $F$-algebra of degree $p$ and let $a_{1}, a_{2}, \ldots, a_{n}$ (where $n \geq 1$ ) be a sequence of elements in $F^{\times}$. We define a standard norm variety $W\left(L, a_{1}, a_{2}, \ldots, a_{n}\right)$ for the sequence $\left(L, a_{1}, a_{2}, \ldots, a_{n}\right)$ inductively as follows. Let $W\left(L, a_{1}\right)$ be the Severi-Brauer variety for the cyclic algebra $\left(L / F, a_{1}\right)$ of degree $p$ and for $n>1$ let $W\left(L, a_{1}, a_{2}, \ldots, a_{n}\right)$ be a smooth compactification of the smooth variety $V\left(W\left(L, a_{1}, a_{2}, \ldots, a_{n-1}\right), a_{n}\right)$. Note that $W\left(L, a_{1}, a_{2}, \ldots, a_{n}\right)$ is a smooth projective geometrically irreducible variety over $F$ of dimension $p^{n}-1$. Note that the birational class of a standard norm variety $W\left(L, a_{1}, a_{2}, \ldots, a_{n}\right)$ is uniquely determined by the sequence $\left(L, a_{1}, a_{2}, \ldots, a_{n}\right)$.

Note that if $F$ contains a primitive $p$ th root of unity $\xi_{p}$, we have $L=F\left(a_{0}^{1 / p}\right)$ for some $a_{0} \in F^{\times}$and $W\left(L, a_{1}, a_{2}, \ldots, a_{n}\right)=W\left(a_{0}, a_{1}, a_{2}, \ldots, a_{n}\right)$ as defined in [28, §2].

For small $n$ one can identify the standard norm varieties varieties as follows (see [24] or [23]):

Example 5.6. By definition, $W\left(L, a_{1}\right)$ is the Severi-Brauer variety for the cyclic algebra $A=\left(L / F, a_{1}\right)$. If $W\left(L, a_{1}\right)_{K}$ has a point over a field extension $K / F$, it is isomorphic to the projective space $\mathbb{P}_{K}^{p-1}$. Hence it is rational over $K$ and therefore $W\left(L, a_{1}\right)$ is $A$-trivial.

Example 5.7. The variety $W\left(L, a_{1}, a_{2}\right)$ is birationally isomorphic to the $\mathbf{S L}_{1}(A)$-torsor given by the equation $\mathrm{Nrd}=a_{2}$ in the cyclic algebra $A=\left(L / F, a_{1}\right)$, where $\mathrm{Nrd}$ is the reduced norm map for $A$. If $W\left(L, a_{1}, a_{2}\right)$ has a rational point, it is birationally isomorphic to the variety of the group $\mathbf{S L}_{1}(A)$ and hence is retract rational by Proposition 5.1. It follows that $W\left(L, a_{1}, a_{2}\right)$ is $A$-trivial (see Example 5.2).

A sequence $\left(L, a_{1}, a_{2}, \ldots, a_{n}\right)$ as above determines the symbol

$$
\left\{L, a_{1}, a_{2}, \ldots, a_{n}\right\}:=(L) \cup\left(a_{1}\right) \cup \cdots \cup\left(a_{n}\right)
$$

in $H^{n+1}\left(F, \mu_{p}^{\otimes n}\right)$, where $(L) \in H^{1}(F, \mathbb{Z} / p \mathbb{Z})$ and $\left(a_{i}\right) \in H^{1}\left(F, \mu_{p}\right)$ are the obvious classes. By [28], the standard norm variety of the sequence $\left(L, a_{1}, a_{2}, \ldots, a_{n}\right)$ is a norm variety of the symbol $\left\{L, a_{1}, a_{2}, \ldots, a_{n}\right\}$.

Theorem 5.8. Let $F$ be a field of characteristic $0, p$ a prime integer, $L / F$ a cyclic field extension of degree $p$ and $a_{1}, \ldots, a_{n} \in F^{\times}$. If the symbol $\left\{L, a_{1}, a_{2}, \ldots, a_{n}\right\}$ is nontrivial, then a standard norm variety $X:=W\left(L, a_{1}, a_{2}, \ldots, a_{n}\right)$ is A-trivial for the coefficient ring $\Lambda=\mathbb{Z}_{(p)}$. Moreover, the degree map deg: $\mathrm{CH}_{0}(X) \rightarrow \mathbb{Z}_{(p)}$ is injective. 
Proof. In the proof we may assume that $n \geq 2$ (see Example 5.6). We induct on $n$. Write for simplicity

$$
Y=W\left(L, a_{1}, a_{2}, \ldots, a_{n-1}\right) \text { and } U=V\left(Y, a_{n}\right) .
$$

Thus, $X$ is a smooth compactification of $U$.

Claim: Let $K / F$ be a field extension such that the symbol $\left\{L, a_{1}, \ldots, a_{n-1}\right\}$ is nontrivial and $x_{1}, x_{2} \in U(K)$. Then $\left[x_{1}\right]=\left[x_{2}\right]$ in $\mathrm{CH}_{0}(X)$ (with the coefficient ring $\Lambda=\mathbb{Z}_{(p)}$ ).

Replacing $F$ by $K$, we may assume that $K=F$. Moreover, we can also assume that $F$ is $p$-special, in particular, $F$ contains $\xi_{p}$. We shall write $\left\{a_{0}, a_{1}, \ldots, a_{n-1}\right\}$ for $\left\{L, a_{1}, \ldots, a_{n-1}\right\}$.

Let $E_{i}=F\left\{z_{i}\right\}, i=1,2$, where $z_{i}$ is the image of $x_{i}$ under the morphism $U \rightarrow \widetilde{S}^{p}(Y)$. Each $E_{i}$ is a cyclic field extension of $F$ of degree $p$. By Lemma 5.5, $Y\left(E_{i}\right) \neq \emptyset$, and hence $E_{i}$ splits the symbol $\left\{a_{0}, a_{1}, \ldots, a_{n-1}\right\}$ as $Y$ is a splitting variety for this symbol. By $[28$, Theorem 5.6], there are $b_{0}, b_{1} \in F^{\times}$such that the symbol $\left\{b_{0}, b_{1}\right\}$ divides $\left\{a_{0}, a_{1}, \ldots, a_{n-1}\right\}$ and both $E_{i}$ split $\left\{b_{0}, b_{1}\right\}$. Let $Y^{\prime}$ be the Severi-Brauer variety for the cyclic algebra $\left(F\left(b_{0}^{1 / p}\right) / F, b_{1}\right)$. Let $U^{\prime}=V\left(Y^{\prime}, a_{n}\right)$ and let $X^{\prime}$ be a smooth compactification of $U^{\prime}$, i.e. $X^{\prime}$ is a standard norm variety for the 3 -sequence $\left(b_{0}, b_{1}, a_{n}\right)$.

The function field $F\left(Y^{\prime}\right)$ splits the symbol $\left\{b_{0}, b_{1}\right\}$ and hence also splits $\left\{a_{0}, a_{1}, \ldots, a_{n-1}\right\}$. As $Y$ is a $p$-generic splitting variety of the symbol $\left\{a_{0}, a_{1}, \ldots, a_{n-1}\right\}$, there is a finite field extension $M / F\left(Y^{\prime}\right)$ of degree prime to $p$ such that $Y$ has a point over $M$. Choose a smooth projective model $Y^{\prime \prime}$ of $M$ over $F$. There are two rational maps

$$
Y^{\prime} \leftrightarrow-Y^{\prime \prime} \rightarrow Y \text {, }
$$

where the left map is dominant of degree prime to $p$. The right one is not constant as $Y$ has no $F$-point. Therefore, the image of the induced rational map $S^{p}\left(Y^{\prime \prime}\right) \rightarrow S^{p}(Y)$ intersects $\widetilde{S}^{p}(Y)$ nontrivially and hence we have two rational maps $\widetilde{R}_{E_{i} / F}\left(Y_{E_{i}}^{\prime \prime}\right) \rightarrow-\widetilde{R}_{E_{i} / F}\left(Y_{E_{i}}\right)$ (for $i=1,2)$. Thus, there are two commutative diagrams:

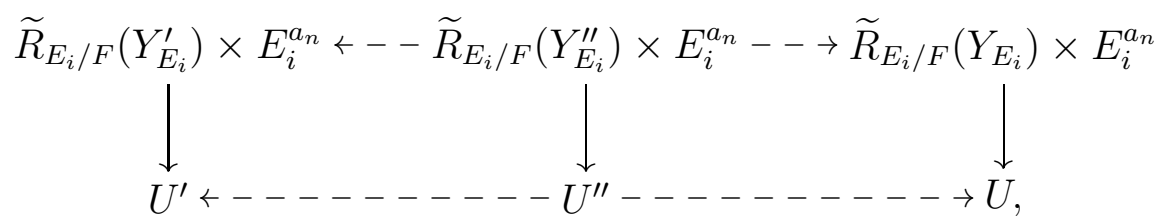

where $U^{\prime \prime}=V\left(Y^{\prime \prime}, a_{n}\right)$.

The variety $E_{i}^{a_{n}}$ is a torsor under the norm one torus $T_{i}$ for the cyclic extension $E_{i} / F$ of degree $p$. As $T_{i} \simeq R_{E_{i} / F}\left(\mathbb{G}_{m, E_{i}}\right) / \mathbb{G}_{m}$ and $E_{i}^{a_{n}}$ has a rational point, we can embed the variety $E_{i}^{a_{n}} \simeq T_{i}$ as an open subset into the projective space $\mathbb{P}\left(E_{i}\right)$. Thus, we have the following commutative diagram of rational maps of smooth projective varieties:

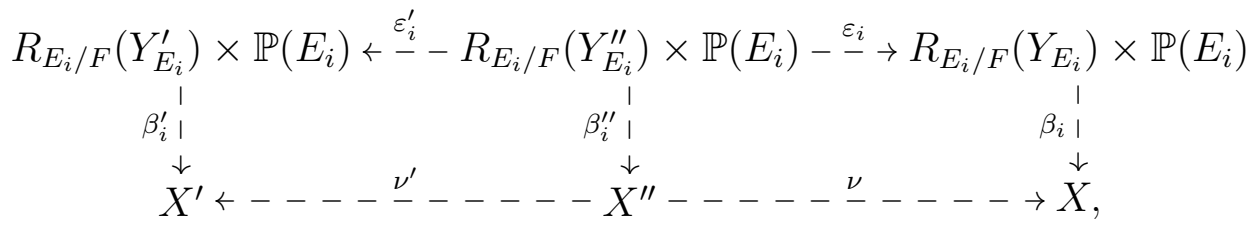

where $X^{\prime \prime}$ is a smooth compactification of $U^{\prime \prime}$. 
By Proposition RC.14, we have the equality $\left(\nu^{\prime}\right)^{t} \circ \beta_{i}^{\prime}=\beta_{i}^{\prime \prime} \circ\left(\varepsilon_{i}^{\prime}\right)^{t}$ of rational correspondences. Hence we get a commutative diagram of rational correspondences between smooth projective varieties:

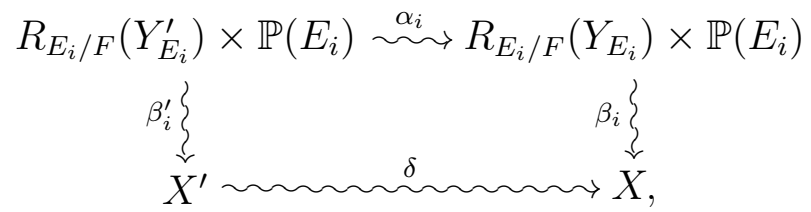

where $\alpha_{i}=\varepsilon_{i} \circ\left(\varepsilon_{i}^{\prime}\right)^{t}$ and $\delta=\nu \circ\left(\nu^{\prime}\right)^{t}$.

Note that the correspondences $\beta$ and $\beta_{i}$ are of multiplicity 1 and the correspondences $\alpha_{i}$ and $\delta$ are of multiplicity prime to $p$.

Choose rational points $w_{i}$ in $R_{E_{i} / F}\left(Y_{E_{i}}^{\prime}\right) \times \mathbb{P}\left(E_{i}\right)$. As $X^{\prime}$ is $A$-trivial by Example 5.7 and the 0 -cycles $\beta_{i_{*}}^{\prime}\left(\left[w_{i}\right]\right)$ both have degree 1 , we have $\beta_{1_{*}}^{\prime}\left(\left[w_{1}\right]\right)=\beta_{2 *}^{\prime}\left(\left[w_{2}\right]\right)$ in $\mathrm{CH}_{0}\left(X^{\prime}\right)$. It follows that

$$
\beta_{1 *}\left(\alpha_{1 *}\left(\left[w_{1}\right]\right)\right)=\delta_{*}\left(\beta_{1 *}^{\prime}\left(\left[w_{1}\right]\right)\right)=\delta_{*}\left(\beta_{2 *}^{\prime}\left(\left[w_{2}\right]\right)\right)=\beta_{2 *}\left(\alpha_{2 *}\left(\left[w_{2}\right]\right)\right) .
$$

By Lemma 5.5, there are rational points $y_{i}$ in $\widetilde{R}_{E_{i} / F}\left(Y_{E_{i}}\right) \times E^{a_{n}}$ over $x_{i}$. It follows from Lemma RC.10 that $\beta_{i_{*}}\left(\left[y_{i}\right]\right)=\left[x_{i}\right]$ in $\mathrm{CH}_{0}(X)$.

Since by the induction hypothesis $Y$ is $A$-trivial, it follows from Proposition 5.4 that the varieties $R_{E_{i} / F}\left(Y_{E_{i}}\right) \times \mathbb{P}\left(E_{i}\right)$ are $A$-trivial, hence

$$
\alpha_{i *}\left(\left[w_{i}\right]\right)=m\left[y_{i}\right]
$$

where $m=\operatorname{mult}(\delta)=\operatorname{mult}\left(\alpha_{i}\right)=\operatorname{deg} \alpha_{i *}\left(\left[w_{i}\right]\right)$. It follows from (5.9) and (5.10) that

$$
m\left[x_{1}\right]=m \beta_{1 *}\left(\left[y_{1}\right]\right)=\beta_{1 *}\left(\alpha_{1 *}\left(\left[w_{1}\right]\right)\right)=\beta_{2 *}\left(\alpha_{2 *}\left(\left[w_{2}\right]\right)\right)=m \beta_{2 *}\left(\left[y_{2}\right]\right)=m\left[x_{2}\right]
$$

in $\mathrm{CH}_{0}(X)$. Since $m$ is prime to $p$, the claim is proved.

Now we finish the proof of the first part of the theorem. Let $K / F$ be a field extension and $x_{1}, x_{2} \in X(K)$. By Proposition 5.3, it suffices to show that $\left[x_{1}\right]=\left[x_{2}\right]$ in $\mathrm{CH}_{0}\left(X_{K}\right)$.

Consider the field $L=F(X \times X)$ and the two "generic" points $\xi_{1}$ and $\xi_{2}$ in $X(L)$. Note that $\xi_{i} \in U(L)$ and the field $F(X \times X)$ does not split the symbol $\left\{a_{1}, \ldots, a_{n-1}\right\}$ : otherwise the variety $Y$ would be equivalent (in the sense of Section 3) to the varieties $X \sim X \times X$ and we would have $\operatorname{cd}_{p} Y=\operatorname{cd}_{p} X$ by Lemma 3.6 contradicting $\operatorname{cd}_{p} Y=$ $p^{n-1}-1<p^{n}-1=\operatorname{cd}_{p} X$ (see Corollary 3.9). By the claim, $\left[\xi_{1}\right]=\left[\xi_{2}\right]$ in $\mathrm{CH}_{0}\left(X_{L}\right)$. Specializing $\xi_{i}$ to $x_{i}$ (see [9, §20.3] or [27]), we get the result.

We prove now that the degree map deg $: \mathrm{CH}_{0}(X) \rightarrow \mathbb{Z}_{(p)}$ is injective. We may assume that $F$ is a $p$-special field. We claim that if $x$ and $x^{\prime}$ are two closed points of $X$ of degree $p$, then $[x]=\left[x^{\prime}\right]$ in $\mathrm{CH}_{0}(X)$. To prove the claim, as in the first part of the proof, we find a Severi-Brauer variety $Y$ over $F$, that is split by the two field extensions $L:=F(x)$ and $L^{\prime}:=F\left(x^{\prime}\right)$ of $F$, and a correspondence $\delta: Y \rightsquigarrow X$ of degree $m$ prime to $p$. Let $y$ and $y^{\prime}$ be closed points of $Y$ with residue fields isomorphic to $L$ and $L^{\prime}$ respectively.

Consider the correspondence $\delta_{L}: Y_{L} \rightsquigarrow X_{L}$ and rational points $y_{1} \in Y_{L}, x_{1} \in X_{L}$ over $y$ and $x$ respectively. As $\delta_{*}\left(\left[y_{1}\right]\right)$ is a 0 -cycle on $X_{L}$ of degree $m$ and the variety $X$ is $A$-trivial by the first part of the proof, we have $\left(\delta_{L}\right)_{*}\left(\left[y_{1}\right]\right)=m\left[x_{1}\right]$. Taking the norms for the extension $L / F$, we get $\delta_{*}([y])=m[x]$ in $\mathrm{CH}_{0}(X)$. Similarly, $\delta_{*}\left(\left[y^{\prime}\right]\right)=m\left[x^{\prime}\right]$. As $[y]=\left[y^{\prime}\right]$ in $\mathrm{CH}_{0}(Y)$ by [25 or [22], we have $[x]=\left[x^{\prime}\right]$ in $\mathrm{CH}_{0}(X)$. The claim is proved. 
Take any closed point $x \in X$ and set $\operatorname{deg}(x)=p^{k}$ for some $k>0$. As $\operatorname{deg} \mathrm{CH}_{0}(X)=$ $p \mathbb{Z}_{(p)}$ and $F$ is $p$-special, there is a closed point $x^{\prime} \in X$ of degree $p$. It suffices to show that $[x]=p^{k-1}\left[x^{\prime}\right]$ in $\mathrm{CH}_{0}(X)$. Choose a field $K$ with $F \subset K \subset F(x)$ and $[F(x): K]=p$. Let $x_{1}$ be a closed point in $X_{K}$ over $x$ with $\operatorname{deg}\left(x_{1}\right)=p$. We shall show that $\left[x_{1}\right]=\left[x^{\prime}\right]_{K}$ in $\mathrm{CH}_{0}\left(X_{K}\right)$ and then taking the norms for the extension $K / F$ we get the desired equality. As both cycles $\left[x_{1}\right]$ and $\left[x^{\prime}\right]_{K}$ have degree $p$, they are equal in the case $X(K) \neq \emptyset$ as $X$ is $A$-trivial. In the case $X(K)=\emptyset$ the cycles $\left[x_{1}\right]$ and $\left[x^{\prime}\right]_{K}$ are equal by the claim as $x_{1}$ and $x_{K}^{\prime}$ are points in $X_{K}$ of degree $p$.

\section{Appendix RC. Rational CORRESpondences}

We review the construction of the category of rational correspondences due to M. Rost.

RC-I. Integral correspondences. Let $M$ be a cycle module over a field $F$ and let $X$ be an algebraic variety over $F$. The groups

$$
C_{p}(X ; M)=\coprod_{x \in X_{(p)}} M(x),
$$

where $M(x)=M(F(x))$, form a cycle complex $C(X ; M)$ [27, 3.2]. Denote by $A_{p}(X ; M)$ the homology groups of $C(X ; M)$. If $X$ is equidimensional of dimension $d_{X}$ we set

$$
A^{q}(X ; M)=A_{d_{X}-q}(X ; M) .
$$

Example RC.1. If $M=K$ is given by Milnor's $K$-theory of fields, $A_{p}(X ; K)$ are the Milnor $K$-cohomology groups of $X$, in particular, the Chow groups of $X$ (see [7, Chapter IX]).

Let $X, Y$ and $Z$ be algebraic varieties over $F$ with $X$ irreducible smooth and complete. We define a pairing $(\cup$-product $)$

$$
\mathrm{CH}_{r}(X \times Y) \otimes A_{p}(Z \times X ; M) \stackrel{\cup}{\rightarrow} A_{r+p-d_{X}}(Z \times Y ; M), \quad(v, a) \mapsto v \cup a
$$

as the composition

$$
\begin{aligned}
& \mathrm{CH}_{r}(X \times Y) \otimes A_{p}(Z \times X ; M) \stackrel{\times}{\rightarrow} A_{r+p}(X \times X \times Z \times Y ; M) \\
& \stackrel{\left(\Delta \times \operatorname{id}_{Y \times Z}\right)^{*}}{\longrightarrow} A_{r+p-d_{X}}(X \times Z \times Y ; M) \stackrel{q_{*}}{\rightarrow} A_{r+p-d_{X}}(Z \times Y ; M),
\end{aligned}
$$

where $\times$ is the external product, $\Delta: X \rightarrow X \times X$ is the diagonal embedding and $q: X \times Z \times Y \rightarrow Z \times Y$ is the projection. (See 27 for the definitions. We need $X$ smooth to get $\Delta$ a regular embedding and $X$ complete to have $q$ proper.)

Example RC.2. Let $f: X \rightarrow Y$ and $g: Y \rightarrow X$ be morphisms with the graphs $\Gamma_{f} \subset X \times Y$ and $\Gamma_{g} \subset Y \times X$. Then $\left[\Gamma_{f}\right] \cup=\left(\operatorname{id}_{Z} \times f\right)_{*}$ and $\left[\Gamma_{g}\right]^{t} \cup=\left(\operatorname{id}_{Z} \times g\right)^{*}$ (here $t$ is the transposition involution).

In particular, we have the product

$$
\mathrm{CH}_{r}(X \times Y) \otimes \mathrm{CH}_{p}(Z \times X) \stackrel{\cup}{\rightarrow} \mathrm{CH}_{r+p-d_{X}}(Z \times Y) .
$$


It is taken as the composition law for the category of integral correspondences $\operatorname{Cor}(F)$ (see [17] and [7, Chapter XII]) with the objects smooth complete varieties over $F$ and morphisms

$$
\operatorname{Mor}_{\mathrm{Cor}(F)}(X, Y)=\coprod_{i} \mathrm{CH}_{d_{i}}\left(X_{i} \times Y\right),
$$

where $X_{i}$ are irreducible (connected) components of $X$ with $d_{i}=\operatorname{dim} X_{i}$.

Denote by $\operatorname{Var}(F)$ the category of smooth complete varieties over $F$ and morphisms of varieties. There is a natural functor

$$
\operatorname{Var}(F) \rightarrow \operatorname{Cor}(F), \quad X \mapsto X, \quad f \mapsto\left[\Gamma_{f}\right] .
$$

The functors $\operatorname{Var}(F) \rightarrow \mathrm{Ab}, X \mapsto A_{p}(X ; M)$ and $X \mapsto A^{p}(X ; M)$ factor through a covariant functor $\operatorname{Cor}(F) \rightarrow \mathrm{Ab}$,

$$
X \mapsto A_{p}(X ; M), \quad a \mapsto a \cup
$$

and a contravariant functor

$$
X \mapsto A^{p}(X ; M), \quad a \mapsto a^{t} \cup
$$

RC-II. The cycle module $A_{0}[Y, M]$. For a cycle module $M$ over $F$ and an algebraic variety $Y$ over $F$ define the cycle module $A_{0}[Y, M]$ over $F$ by

$$
A_{0}[Y, M](L)=A_{0}\left(Y_{L}, M\right)
$$

(see [27, §7]). There is a canonical map of complexes

$$
\theta_{Y, M}: C(X \times Y ; M) \rightarrow C\left(X ; A_{0}[Y, M]\right),
$$

that takes an element in $M(z)$ for $z \in(X \times Y)_{(p)}$ to zero if dimension of the projection $x$ of $z$ in $X$ is less than $p$ and identically to itself otherwise. In the latter case we consider $z$ as a point of dimension 0 in $Y_{x}:=Y_{F(x)}$ under the inclusion $Y_{x} \subset X \times Y$. Thus, $\theta_{Y, M}$ "ignores" points in $X \times Y$ that lose dimension being projected to $X$.

We study various compatibility properties of $\theta$.

RC-II.1. Cross products. Let $N \times M \rightarrow P$ be a bilinear pairing of cycle modules over $F$. For a variety $Y$ over $F$ we can define a pairing

$$
A_{0}[Y, N] \times M \rightarrow A_{0}[Y, P]
$$

in an obvious way.

Lemma RC.5. Let $M$ be a cycle module, $X, Y$ and $Z$ varieties over $F$. Then the following diagram is commutative:

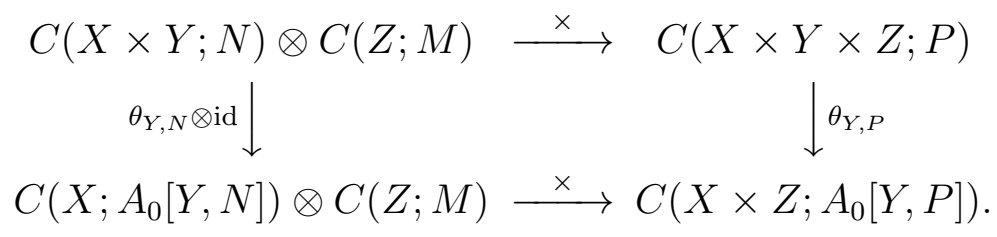


Proof. Let $z \in Z_{(k)}$ and $\mu \in C(Z ; M)$. Consider the following commutative diagram

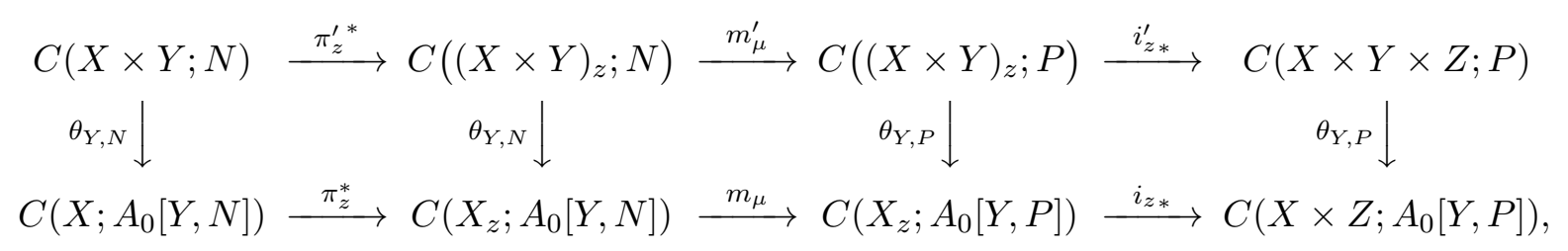
where $\pi_{z}: X_{z} \rightarrow X$ and $\pi_{z}^{\prime}:(X \times Y)_{z} \rightarrow X \times Y$ are the natural projections, $m_{\mu}$ and $m_{\mu}^{\prime}$ are the multiplications by $\mu, i_{z}: X_{z} \rightarrow X \times Z$ and $i_{z}^{\prime}:(X \times Y)_{z} \rightarrow X \times Y \times Z$ are the inclusions. By the definition of the cross product, the compositions in the two rows of the diagram are the multiplications by $\mu$.

RC-II.2. Pull-back maps. Let $f: Z \hookrightarrow X$ be a regular closed embedding, $N_{X / Z}$ the normal bundle over $Z$. Choose a coordination $\tau$ of $N_{X / Z}$ [27, §9]. For a variety $Y$, the closed embedding

$$
f^{\prime}=f \times \operatorname{id}_{Y}: Z \times Y \hookrightarrow X \times Y
$$

is also regular and the normal bundle $N_{X \times Y / Z \times Y}$ is isomorphic to $N_{X / Z} \times Y$. We choose the induced coordination $\tau^{\prime}=\tau \times Y$ of $N_{X \times Y / Z \times Y}$.

Lemma RC.6. For every cycle module $M$ the following diagram commutes:

$$
\begin{array}{ccc}
C(X \times Y ; M) \stackrel{I\left(f^{\prime}\right)}{\longrightarrow} & C(Z \times Y ; M) \\
\theta_{Y, M} \downarrow & \downarrow \theta_{Y, M} \\
C\left(X ; A_{0}[Y, M]\right) \stackrel{I(f)}{\longrightarrow} C\left(Z ; A_{0}[Y, M]\right),
\end{array}
$$

where the pull-back maps $I(f)$ and $I\left(f^{\prime}\right)$ are chosen with respect to the coordinations $\tau$ and $\tau^{\prime}$ respectively (see [27, §12]).

Proof. Let $q: X \times \mathbb{G}_{m} \rightarrow X$ and $q^{\prime}: X \times Y \times \mathbb{G}_{m} \rightarrow X \times Y$ be the natural projections. The following diagram is clearly commutative:

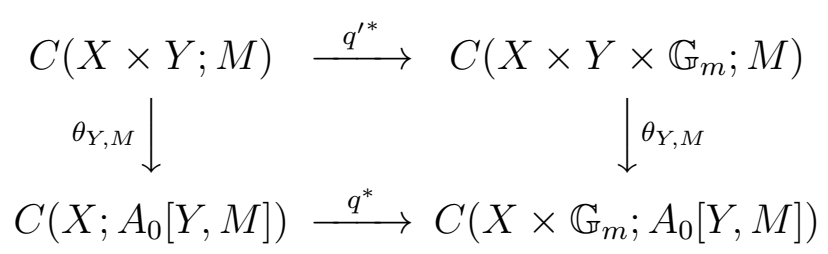

(here $q^{*}$ and $q^{\prime *}$ are the flat pull-back maps [27, 3.5]).

Let $t$ be the coordinate function on $\mathbb{G}_{m}$. The map $\theta_{Y, M}$ clearly commutes with the multiplication by $t$, i.e. the following diagram is commutative:

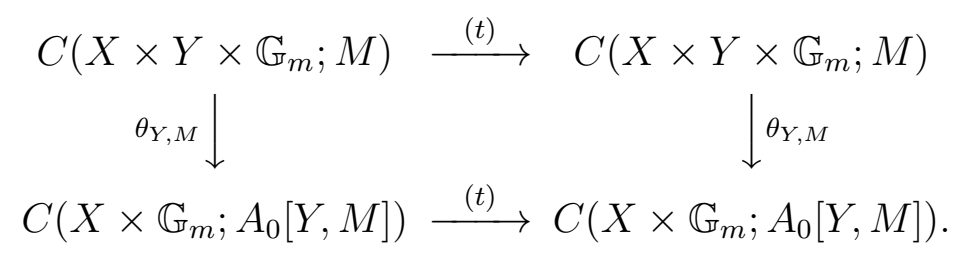

Let $D=D(X, Z)$ be the deformation space of the embedding $f$ [27, $\S 10]$. There is a closed embedding $i: N_{X / Z} \hookrightarrow D$ with the open complement $j: X \times \mathbb{G}_{m} \hookrightarrow D$. Then 
$D^{\prime}=D \times Y$ is the deformation space $D(X \times Y, Z \times Y)$ with the closed embedding

$$
i^{\prime}=i \times \operatorname{id}_{Y}: N_{X \times Y / Z \times Y} \hookrightarrow D^{\prime}
$$

and the open complement $j^{\prime}=j \times \operatorname{id}_{Y}: X \times Y \times \mathbb{G}_{m} \hookrightarrow D^{\prime}$.

The commutative diagram of complexes with the exact rows

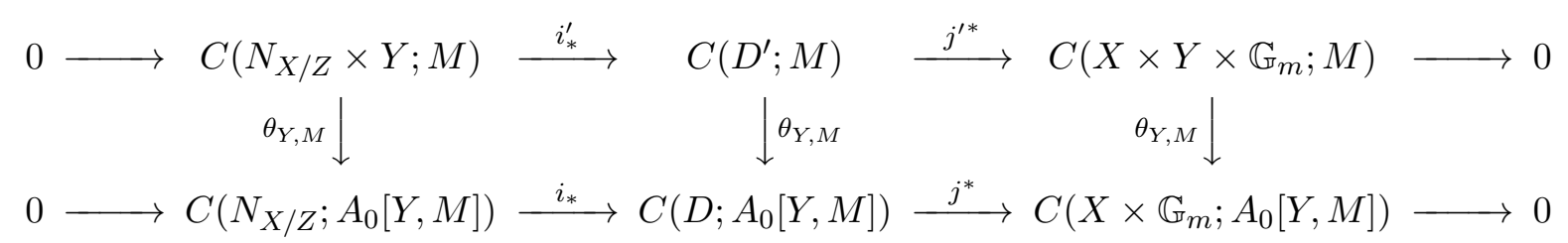

induces the commutative diagram

$$
\begin{array}{ccc}
C\left(X \times Y \times \mathbb{G}_{m} ; M\right) & \partial & C\left(N_{X / Z} \times Y ; M\right) \\
\theta_{Y, M} \downarrow & \downarrow \theta_{Y, M} \\
C\left(X \times \mathbb{G}_{m} ; A_{0}[Y, M]\right) \stackrel{\partial}{\longrightarrow} C\left(N_{X / Z} ; A_{0}[Y, M]\right) .
\end{array}
$$

The coordinations $\tau$ and $\tau^{\prime}$ induce the commutative diagram [27, §9]

$$
\begin{array}{ccc}
C\left(N_{X / Z} \times Y ; M\right) & \stackrel{r\left(\tau^{\prime}\right)}{\longrightarrow} & C(Z \times Y ; M) \\
\theta_{Y, M} \downarrow & \downarrow \theta_{Y, M} \\
C\left(N_{X / Z} ; A_{0}[Y, M]\right) \stackrel{r(\tau)}{\longrightarrow} C\left(Z ; A_{0}[Y, M]\right) .
\end{array}
$$

By the definition of the pull-back map, the diagram in question is the composition of the four commutative square diagrams considered in the proof.

RC-II.3. Push-forward maps. Let $f: X \rightarrow Z$ be a morphism of varieties over $F$. For a variety $Y$ set

$$
f^{\prime}=f \times \operatorname{id}_{Y}: X \times Y \rightarrow Z \times Y .
$$

Lemma RC.7. The following diagram is commutative:

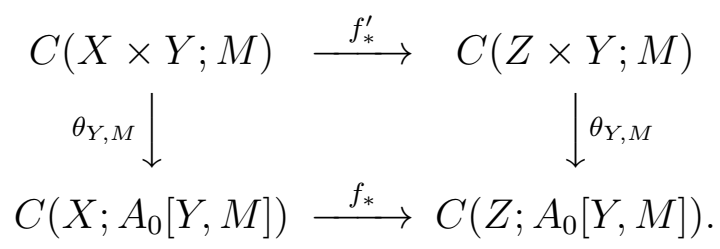

Proof. Let $u \in(X \times Y)_{(p)}, a \in M(u)$. Set $v=f^{\prime}(u) \in Z \times Y$. If $\operatorname{dim}(v)<p$ then $\left(f_{*}^{\prime}\right)_{u}(a)=0$. In this case the dimension of the projection $y$ of $u$ in $Y$ is less than $p$ and hence $\theta_{u}(a)=0$.

Assume that $\operatorname{dim}(v)=p$. Then $F(u) / F(v)$ is a finite field extension and

$$
b=\left(f_{*}^{\prime}\right)_{u}(a)=c_{F(u) / F(v)}(a) \in M(v),
$$

where $c_{F(u) / F(v)}$ is the norm map. If $\operatorname{dim}(y)<p$, then $\theta_{u}(a)=0$ and $\theta_{v}(b)=0$.

Assume that $\operatorname{dim}(y)=p$. Then

$$
\left(\theta \circ f_{*}^{\prime}\right)_{u}(a)=c_{F(u) / F(v)}(a)=b
$$


considered as an element of $A_{0}[Y ; M](z)=A_{0}\left(Y_{z} ; M\right)$, where $z$ is the image of $v$ in $Z$. On the other hand,

$$
\left(f_{*} \circ \theta\right)_{u}(a)=\varphi_{*}(a),
$$

where $\varphi: Y_{x} \rightarrow Y_{z}$ is the natural morphism ( $x$ is the image of $u$ in $X$ ) and $a$ is considered as an element of $A_{0}[Y ; M](x)$. It remains to notice that

$$
\varphi_{*}(a)=c_{F(u) / F(v)}(a)=b .
$$

RC-III. The category of rational correspondences. Let $X$ and $Y$ be varieties over $F$ and let $M$ be a cycle module over $F$. By Lemma RC.5, for the pairing $K \times M \rightarrow M$ and $Z=X$ we have the commutative diagram

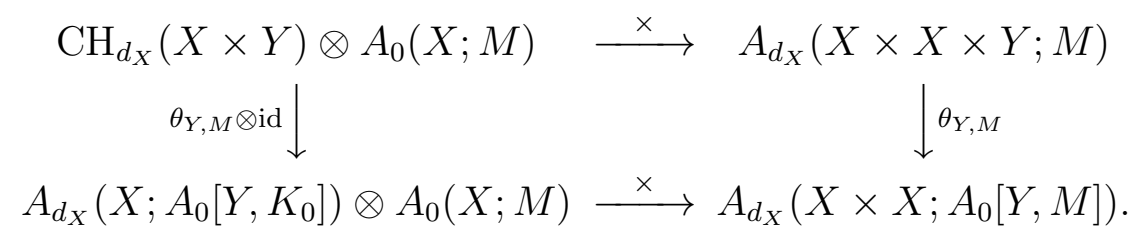

Assume that $X$ is smooth. Let $\Delta: X \rightarrow X \times X$ be the diagonal embedding and $\Delta^{\prime}=\Delta \times \operatorname{id}_{Y}$. By Lemma RC.6, the following diagram is commutative:

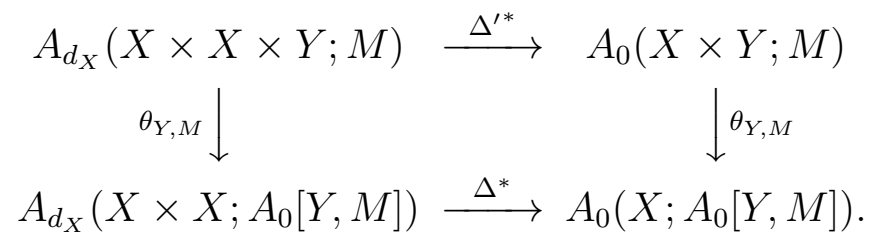

Finally, assume that $X$ is complete. Let $f: X \rightarrow \operatorname{Spec} F$ be the structure morphism and $f^{\prime}=f \times \operatorname{id}_{Y}$. Lemma RC.7 gives the following commutative diagram:

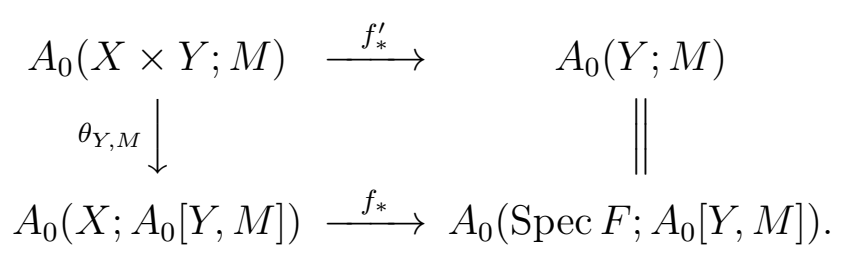

Proposition RC.8. Let $X$ and $Y$ be varieties over $F$, let $X$ be irreducible smooth and proper and let $M$ be a cycle module over $F$. Then the pairing

$$
\mathrm{CH}_{d_{X}}(X \times Y) \otimes A_{0}(X ; M) \stackrel{\cup}{\rightarrow} A_{0}(Y ; M)
$$

is trivial on all cycles in $\mathrm{CH}_{d_{X}}(X \times Y)$ that are not dominant over $X$. In other words, the $\cup$-product factors through a natural pairing

$$
r: \mathrm{CH}_{0}\left(Y_{F(X)}\right) \otimes A_{0}(X ; M) \stackrel{\cup}{\rightarrow} A_{0}(Y ; M) .
$$

Proof. Composing all three diagrams in Section RC-III and taking into account that

$$
A_{d_{X}}\left(X ; A_{0}\left[Y, K_{0}\right]\right)=\mathrm{CH}_{0}\left(Y_{F(X)}\right),
$$


we get the commutative diagram

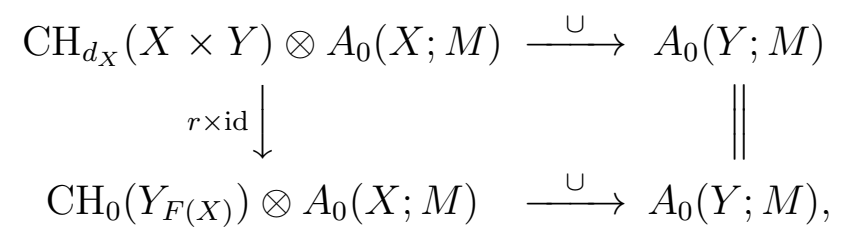

whence the statement.

In the conditions of Proposition RC.8, for an irreducible variety $Z$ over $F$ the diagram

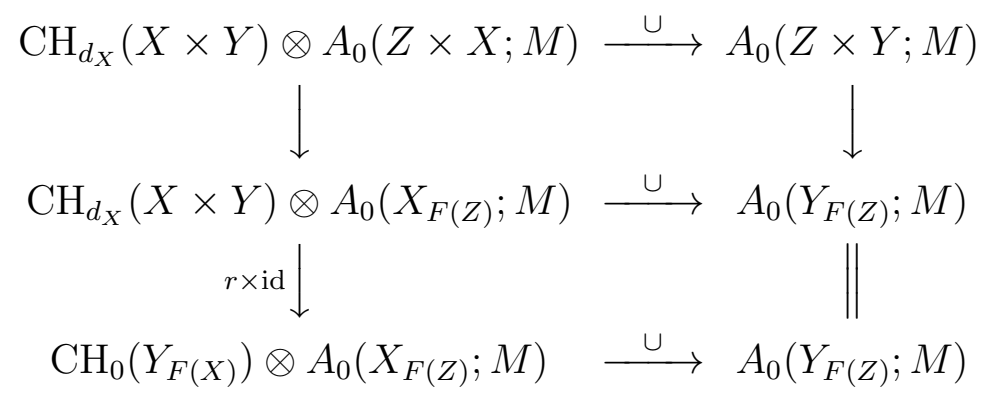

is commutative.

In particular, we have a well defined pairing

$$
\mathrm{CH}_{0}\left(Y_{F(X)}\right) \otimes \mathrm{CH}_{0}\left(X_{F(Z)}\right) \stackrel{\cup}{\rightarrow} \mathrm{CH}_{0}\left(Y_{F(Z)}\right)
$$

that can be taken for the composition law in the category of rational correspondences RatCor $(V)$ with the objects smooth complete varieties over $F$ and the morphisms

$$
\operatorname{Mor}_{\text {RatCor }(F)}(X, Y)=\coprod_{i} \mathrm{CH}_{0}\left(Y_{F\left(X_{i}\right)}\right)
$$

where $X_{i}$ are all irreducible (connected) components of $X$.

There is an obvious functor $\kappa: \operatorname{Cor}(F) \rightarrow \operatorname{Rat} \operatorname{Cor}(F)$.

Theorem RC.9. For a cycle module $M$ there are well defined:

(1) The covariant functor

$$
\operatorname{Rat} \operatorname{Cor}(F) \rightarrow \mathrm{Ab}, \quad X \mapsto A_{0}(X ; M), a \mapsto a \cup,
$$

i.e. the functor $(\overline{R C .3})$ factors through $\kappa$ if $p=0$.

(2) The contravariant functor

$$
\operatorname{Rat} \operatorname{Cor}(F) \rightarrow \mathrm{Ab}, \quad X \mapsto A^{0}(X ; M), a \mapsto a^{t} \cup,
$$

i.e. the functor (RC.4) factors through $\kappa$ if $p=0$.

Proof. The first statement follows from Proposition RC.8. To prove the second part consider an irreducible variety $Y$ and an open subset $j: U \hookrightarrow Y$. For a smooth complete $X$ in the commutative diagram

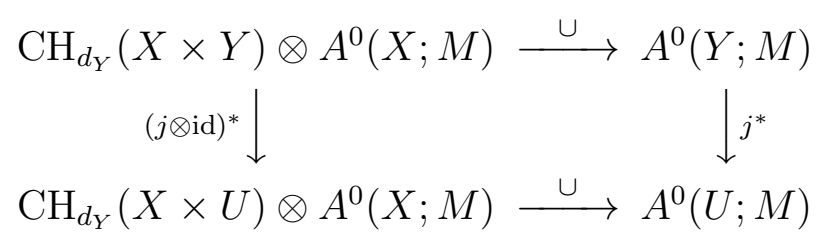


the right vertical homomorphism is injective. Hence the pairing in the top row of the diagram is trivial on the cycles in $\mathrm{CH}_{d_{Y}}(X \times Y)$ that are not dominant over $Y$. Thus we have a well defined pairing

$$
\mathrm{CH}_{d_{Y}}\left(X_{F(Y)}\right) \otimes A^{0}(X ; M) \stackrel{\cup}{\rightarrow} A^{0}(Y ; M)
$$

that defines a contravariant functor

$$
X \mapsto A^{0}(X ; M), \quad a \mapsto a^{t} \cup
$$

By Theorem RC.9, for a cycle module $M$ and a rational correspondence $\alpha: X \rightsquigarrow Y$, we have the two natural homomorphisms

$$
\alpha_{*}:=\alpha \cup: A_{0}(X ; M) \rightarrow A_{0}(Y ; M)
$$

and

$$
\alpha^{*}:=\alpha^{t} \cup: A^{0}(Y ; M) \rightarrow A^{0}(X ; M) .
$$

Let $f: X \rightarrow Y$ be a rational morphism of irreducible varieties. It defines a rational point of $Y_{F(X)}$ over $F(X)$ and hence a morphism in $\operatorname{Mor}_{\text {RatCor }}(X, Y)$ that we still denote by $f: X \rightsquigarrow Y$. In fact, the rational correspondence $f$ is the image of the class of the graph of $f$ under the natural homomorphism $\mathrm{CH}_{d_{X}}(X \times Y) \rightarrow \mathrm{CH}_{0}\left(Y_{F(X)}\right)$.

Lemma RC.10. Let $f: X \rightarrow Y$ be a rational morphism of smooth complete varieties and let $x \in X$ be a rational point such that $f(x)$ is defined. Then $f_{*}([x])=[f(x)]$ in $\mathrm{CH}_{0}(Y)$.

Proof. Let $\Gamma \subset X \times Y$ be the graph of $f$. The preimage of $\{x\} \times \Gamma$ under the morphism $\Delta_{X} \times \mathrm{id}_{Y}: X \times Y \rightarrow X \times X \times Y$ is the reduced scheme $\{x\} \times\{f(x)\}$. It follows from [7, Corollary 57.20] that $\left(\Delta_{X} \times \operatorname{id}_{Y}\right)^{*}([x] \times[\Gamma])=[x] \times[f(x)]$ and hence $f_{*}([x])=$ $q_{*}([x] \times[f(x)])=[f(x)]$, where $q: X \times Y \rightarrow Y$ is the projection.

Corollary RC.11. Let $f: X \rightarrow Y$ and $g: Y \rightarrow Z$ be composable rational morphisms of smooth complete varieties and let $h: X \rightarrow Z$ be the composition of $f$ and $g$. Then $g \circ f=h$ in $\operatorname{Mor}_{\text {RatCor }}(X, Z)$.

Proof. Let $y$ be the rational point of $Y_{F(X)}$ corresponding to $f$. By assumption, the rational morphism $g_{F(X)}: Y_{F(X)} \rightarrow Z_{F(X)}$ is defined at $y$. By Lemma RC.10, the composition of correspondences $f$ and $g$ takes $[y]$ to $\left[g_{F(X)}(y)\right] \in \mathrm{CH}_{0}\left(Z_{F(X)}\right)$. Note that the latter class is given by $h$.

Corollary RC.12. For every two composable rational morphisms $f: X \rightarrow Y$ and $g: Y \rightarrow Z$ of smooth complete varieties, we have $(g \circ f)_{*}=g_{*} \circ f_{*}$ and $(g \circ f)^{*}=f^{*} \circ g^{*}$.

Corollary RC.13 (cf. [27, Corollary 12.10]). The groups $A_{0}(X ; M)$ and $A^{0}(X ; M)$ are birational invariants of the smooth complete variety $X$.

Proposition RC.14. Let

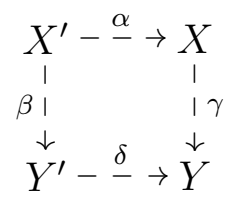


be a commutative diagram of dominant rational morphisms of smooth complete irreducible varieties with $\operatorname{dim}(X)=\operatorname{dim}(Y)$ and $\operatorname{dim}\left(X^{\prime}\right)=\operatorname{dim}\left(Y^{\prime}\right)$. Suppose that the natural ring homomorphism $F(X) \otimes_{F(Y)} F\left(Y^{\prime}\right) \rightarrow F\left(X^{\prime}\right)$ is an isomorphism. Then $\gamma^{t} \circ \delta=\alpha \circ \beta^{t}$.

Proof. The generic fibers of the dominant rational morphisms $\gamma$ and $\beta$ are the single point schemes $\{x\}$ and $\left\{x^{\prime}\right\}$ respectively. We have the following diagram:

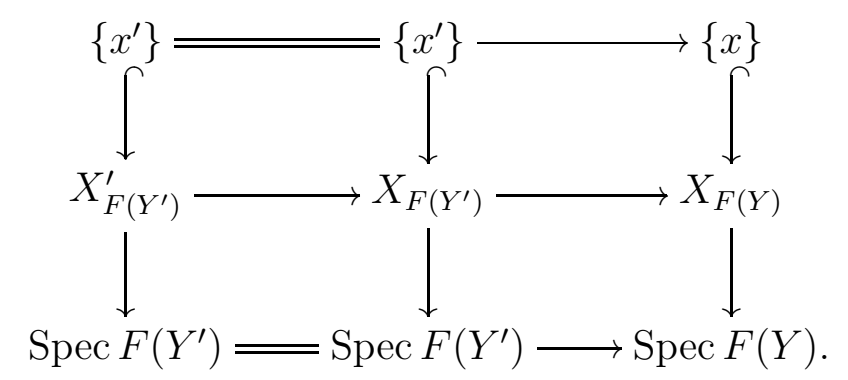

Note that as schemes, $\{x\}=\operatorname{Spec} F(X)$ and $\left\{x^{\prime}\right\}=\operatorname{Spec} F\left(X^{\prime}\right)$. It follows from the assumption that the right part of the diagram is cartesian and hence so is the top right square. In particular, $\left\{x^{\prime}\right\}$ is closed in both $X_{F\left(Y^{\prime}\right)}^{\prime}$ and $X_{F\left(Y^{\prime}\right)}$.

By [7, Proposition 62.4(2)], the composition $\gamma^{t} \circ \delta$ is equal to the image of $[x]$ under the pull-back homomorphism $\mathrm{CH}_{0}\left(X_{F(Y)}\right) \rightarrow \mathrm{CH}_{0}\left(X_{F\left(Y^{\prime}\right)}\right)$ and hence is equal to $\left[x^{\prime}\right]$ as $\left\{x^{\prime}\right\}$ is the fiber product of $\{x\}$ and $X_{F\left(Y^{\prime}\right)}$ over $X_{F(Y)}$.

The composition $\alpha \circ \beta^{t}$ is equal to the image of $\left[x^{\prime}\right]$ under the push-forward homomorphism $\alpha_{*}: \mathrm{CH}_{0}\left(X_{F\left(Y^{\prime}\right)}^{\prime}\right) \rightarrow \mathrm{CH}_{0}\left(X_{F\left(Y^{\prime}\right)}\right)$. The rational map $\alpha_{F\left(Y^{\prime}\right)}: X_{F\left(Y^{\prime}\right)}^{\prime} \rightarrow X_{F\left(Y^{\prime}\right)}$ is defined at $x^{\prime}$ and $\alpha_{F\left(Y^{\prime}\right)}\left(x^{\prime}\right)=x^{\prime}$ as $x^{\prime}$ is the closed point in both $X_{F\left(Y^{\prime}\right)}^{\prime}$ and $X_{F\left(Y^{\prime}\right)}$. It follows from Lemma RC.10 that $\alpha_{*}\left(\left[x^{\prime}\right]\right)=\left[x^{\prime}\right]$ in $\mathrm{CH}_{0}\left(X_{F\left(Y^{\prime}\right)}\right)$.

\section{Appendix RM. Chow groups of Rost motives}

We assume that char $F=0$ here.

RM-I. The binary motive. Let $n$ be a positive integer, $p$ a prime integer and $s$ a symbol in $H^{n+1}\left(F, \mu_{p}^{\otimes n}\right)$. Set

$$
\begin{aligned}
& b=\left(p^{n}-1\right) /(p-1)=1+p+\cdots+p^{n-1}, \\
& c=\left(p^{n+1}-1\right) /(p-1)=1+p+\cdots+p^{n}=b p+1=b+p^{n}, \\
& d=p^{n}-1=b(p-1)=c-b-1 .
\end{aligned}
$$

Let $\mathcal{X}$ be the object in the triangulated category of motivic complexes $\mathbf{D M}(F, \mathbb{Z})$ given by the simplicial scheme of a norm variety of $s$. (Sometimes we will write $\mathcal{X}$ as well for the corresponding object in $\mathbf{D M}\left(F, \mathbb{Z}_{(p)}\right)$.) Write $Q_{i}$ for the Milnor operation in the motivic cohomology of bidegree $\left(2 p^{i}-1, p^{i}-1\right)$ (see [33]) and set

$$
\mu=\left(Q_{1} \circ Q_{2} \circ \cdots \circ Q_{n-1}\right)(\delta) \in H^{2 b+1, b}(\mathcal{X}, \mathbb{Z})
$$

and

$$
\gamma=\left(Q_{1} \circ Q_{2} \circ \cdots \circ Q_{n-1} \circ Q_{n}\right)(\delta)= \pm Q_{n-1}(\mu) \in H^{2 c, c-1}(\mathcal{X}, \mathbb{Z}),
$$

where $\delta \in H^{n+2, n}(\mathcal{X}, \mathbb{Z})$ is the element corresponding to the symbol $s$ (see [20]). 
The binary motive $\mathcal{M}$ of $s$ is defined by the exact triangle

$$
\mathcal{X}(b)[2 b] \stackrel{x}{\rightarrow} \mathcal{M} \stackrel{y}{\rightarrow} \mathcal{X} \stackrel{\mu}{\rightarrow} \mathcal{X}(b)[2 b+1]
$$

in $\mathbf{D M}(F, \mathbb{Z})$.

RM-II. Symmetric powers. As in [33], consider the symmetric powers $\mathrm{S}^{i}(\mathcal{M})$ for $i=$ $1,2, \ldots, p-1$ in $\mathbf{D M}\left(F, \mathbb{Z}_{(p)}\right)$ of the binary motive $\mathcal{M}$. There are the morphisms

$$
a_{i}: \mathrm{S}^{i}(\mathcal{M}) \rightarrow \mathrm{S}^{i-1}(\mathcal{M}) \otimes \mathcal{M} \quad \text { and } \quad b_{i}: \mathrm{S}^{i-1}(\mathcal{M}) \otimes \mathcal{M} \rightarrow \mathrm{S}^{i}(\mathcal{M}),
$$

defined by

$$
a_{i}\left(m_{1} \ldots m_{i}\right)=\sum_{j=1}^{i} m_{1} \ldots \hat{m}_{j} \ldots m_{i} \otimes m_{j} \text { and } b_{i}\left(m_{1} \ldots m_{i-1} \otimes m\right)=m_{1} \ldots m_{i-1} m .
$$

Consider the compositions:

$$
\begin{aligned}
& x_{i}: \mathrm{S}^{i-1}(\mathcal{M})(b)[2 b] \stackrel{1 \otimes x}{\longrightarrow} \mathrm{S}^{i-1}(\mathcal{M}) \otimes \mathcal{M} \stackrel{b_{i}}{\rightarrow} \mathrm{S}^{i}(\mathcal{M}), \\
& y_{i}: \mathrm{S}^{i}(\mathcal{M}) \stackrel{a_{i}}{\rightarrow} \mathrm{S}^{i-1}(\mathcal{M}) \otimes \mathcal{M} \stackrel{1 \otimes y}{\longrightarrow} \mathrm{S}^{i-1}(\mathcal{M}) .
\end{aligned}
$$

We have $x_{1}=x$ and $y_{1}=y$. Set

$$
r_{i}=\mathrm{S}^{i}(y): \mathrm{S}^{i}(\mathcal{M}) \rightarrow \mathcal{X}
$$

so $r_{1}=y$.

The following lemma can be checked by a direct computation:

Lemma RM.1. For every $i=2, \ldots, p-1$,

(1) $y_{i} \circ b_{i-1}-b_{i-2} \circ\left(y_{i-1} \otimes \mathrm{id}_{\mathcal{M}}\right)=\mathrm{id}_{\mathrm{S}^{i-1}(\mathcal{M})} \otimes y$,

(2) $r_{i-1} \circ y_{i}=i \cdot r_{i}$,

(3) $y_{1} y_{2} \ldots y_{i}=i ! \cdot r_{i}$.

Corollary RM.2. The diagram

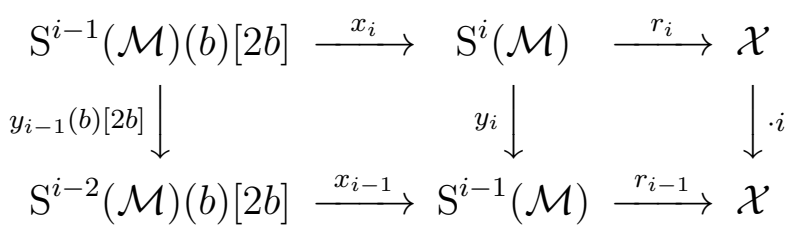

is commutative.

Consider the following objects $\mathcal{S}=\mathrm{S}^{p-2}(\mathcal{M})$ and $\mathcal{R}=\mathrm{S}^{p-1}(\mathcal{M})$ in $\mathbf{D M}\left(F, \mathbb{Z}_{(p)}\right)$. By [33, $\S 5-\S 6$ ], the motive $\mathcal{R}=\mathcal{R}_{s}$ is isomorphic to a Chow motive living on a norm variety of $s$ (this is the only place where we need characteristic 0 ). Since over any field extension of $F$ killing the symbol, the element $\delta$ is trivial, the element $\mu$ is also trivial so that the motive $\mathcal{M}$ is isomorphic to $\mathbb{Z}_{(p)} \oplus \mathbb{Z}_{(p)}(b)$ and the motive $\mathcal{R}$ is isomorphic to the direct sum $\mathbb{Z}_{(p)} \oplus \mathbb{Z}_{(p)}(b) \oplus \cdots \oplus \mathbb{Z}_{(p)}(d)$. It follows that $\mathcal{R}$ is the Rost motive of the symbol $s$ (as defined in Section 四).

Consider the morphism $s=\frac{1}{(p-2) !} y_{2} \ldots y_{p-1}: \mathcal{R} \rightarrow \mathcal{M}$. Taking the compositions of the diagrams in Corollary RM.2 and dividing out $(p-2)$ !, we have: 
Lemma RM.3. The diagram

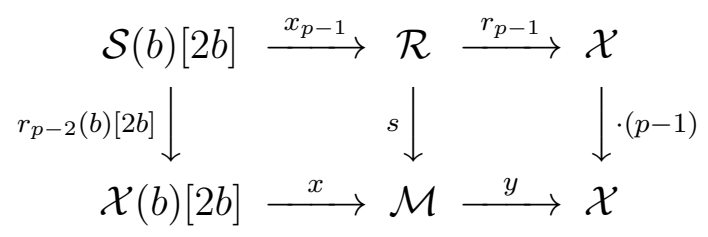

is commutative.

There are exact triangles 33 , (5.5) and (5.6)] in $\mathbf{D M}\left(F, \mathbb{Z}_{(p)}\right)$ :

$$
\begin{gathered}
\mathcal{X}(d)[2 d] \stackrel{\mathrm{S}^{p-1}(x)}{\longrightarrow} \mathcal{R} \stackrel{y_{p-1}}{\longrightarrow} \mathcal{S} \rightarrow \mathcal{X}(d)[2 d+1], \\
\mathcal{S}(b)[2 b] \stackrel{x_{p-1}}{\longrightarrow} \mathcal{R} \stackrel{r_{p-1}}{\longrightarrow} \mathcal{X} \rightarrow \mathcal{S}(b)[2 b+1] .
\end{gathered}
$$

RM-III. Chow groups of Rost motives. For $m \in \mathbb{Z}$, let $K_{m}^{s}(F)$ be the factor group of Milnor's $K$-group $K_{m}(F)$ by the subgroup generated by the images of the norm homomorphisms $K_{m}(L) \rightarrow K_{m}(F)$ over all finite field extensions $L / F$ such that $s$ vanishes over $L$.

By [20, Theorem 1.15], a nontrivial element $\alpha$ of the motivic cohomology group

$$
H^{i, j}(\mathcal{X}, \mathbb{Z}):=H^{i}(\mathcal{X}, \mathbb{Z}(j))
$$

with $i>j$ can be uniquely written in the form

$$
\alpha=x \gamma^{k}\left(Q_{1}^{\varepsilon_{1}} \circ Q_{2}^{\varepsilon_{2}} \circ \cdots \circ Q_{n}^{\varepsilon_{n}}\right)(\delta)=x \gamma^{k} Q^{\varepsilon}(\delta),
$$

where $x \in K_{m}^{s}(F)$ and $k, \varepsilon_{i}$ are integers such that $k \geq 0$ and $\varepsilon_{i}=0$ or 1 . We have

$$
\begin{gathered}
j=m+(c-1) k+\sum \varepsilon_{k}\left(p^{k}-1\right)+n, \\
w(\alpha)=: 2 j-i=m-2 k-|\varepsilon|+(n-2),
\end{gathered}
$$

where $|\varepsilon|=\sum \varepsilon_{k}$.

Note that if $j \leq d$, then $k=0$ and $\varepsilon_{n}=0$.

Lemma RM.7. Let $0 \leq j \leq d$. Then

$$
H^{2 j+1, j}(\mathcal{X}, \mathbb{Z})= \begin{cases}(\mathbb{Z} / p \mathbb{Z}) \mu, & \text { if } j=b ; \\ 0, & \text { otherwise. }\end{cases}
$$

Proof. Let $\alpha=x \cdot Q^{\varepsilon}(\delta) \in H^{2 j+1, j}(\mathcal{X}, \mathbb{Z})$, where $x \in K_{m}^{s}(F)$. Recall that $\varepsilon_{n}=0$, hence $|\varepsilon| \leq n-1$. By (RM.6),

$$
-1=w(\alpha)=m-|\varepsilon|+(n-2)
$$

and therefore,

$$
n-1 \geq|\varepsilon|=m+(n-1) .
$$

It follows that $m=0$ and $Q^{\varepsilon}(\delta)=\left(Q_{1} \circ Q_{2} \circ \cdots \circ Q_{n-1}\right)(\delta)=\mu$.

For every $i=1,2, \ldots, n-1$ set

$$
\widetilde{Q}_{i}=Q_{1} \circ \cdots \circ \widehat{Q}_{i} \circ \cdots \circ Q_{n-1} .
$$

Note that $\widetilde{Q}_{i}(\delta) \in H^{2\left(b-p^{i}+1\right), b-p^{i}+1}(\mathcal{X}, \mathbb{Z})$. 
Lemma RM.8. Let $0 \leq j \leq d$. Then

$$
H^{2 j, j}(\mathcal{X}, \mathbb{Z})= \begin{cases}\mathbb{Z} \cdot 1, & \text { if } j=0 ; \\ (\mathbb{Z} / p \mathbb{Z}) \widetilde{Q}_{i}(\delta), & \text { if } j=b-p^{i}+1 \text { and } 1 \leq i \leq n-1 ; \\ K_{1}^{s}(F) \mu, & \text { if } j=b+1 ; \\ 0, & \text { otherwise. }\end{cases}
$$

Proof. We may assume that $j>0$. Let $\alpha=x Q^{\varepsilon}(\delta) \in H^{2 j, j}(\mathcal{X}, \mathbb{Z})$, where $x \in K_{m}^{s}(F)$. Recall that $|\varepsilon| \leq n-1$. By (RM.6),

$$
0=w(\alpha)=m-|\varepsilon|+(n-2)
$$

and therefore,

$$
n-1 \geq|\varepsilon|=m+(n-2) .
$$

It follows that $m \leq 1$. If $m=0$, we have $|\varepsilon|=n-2$, hence $Q^{\varepsilon}=\widetilde{Q}_{i}$ for $i=1,2, \ldots, n-1$ and $\alpha \in(\mathbb{Z} / p \mathbb{Z}) \widetilde{Q}_{i}(\delta)$.

If $m=1$, then $|\varepsilon|=n-1$ and $Q^{\varepsilon}=Q_{1} \circ Q_{2} \circ \cdots \circ Q_{n-1}$, hence $\alpha \in K_{1}^{s}(F) \mu$.

Lemma RM.9. The canonical map

$$
H^{i, j}\left(\mathcal{S}, \mathbb{Z}_{(p)}\right) \rightarrow H^{i, j}\left(\mathcal{R}, \mathbb{Z}_{(p)}\right)
$$

is an isomorphism if $i<2 d$ and $j<d$.

Proof. Use the triangle (RM.4).

The Chow groups with coefficients in $\mathbb{Z}_{(p)}$ of a motive $\mathcal{N}$ in $\operatorname{DM}\left(F, \mathbb{Z}_{(p)}\right)$ are defined as

$$
\mathrm{CH}^{i}(\mathcal{N}):=H^{2 i, i}\left(\mathcal{N}, \mathbb{Z}_{(p)}\right) \text {. }
$$

Theorem RM.10. Let $\mathcal{R}$ be the Rost motive of a nontrivial $(n+1)$-symbol modulo $p$. Then

$$
\mathrm{CH}^{j}(\mathcal{R})= \begin{cases}\mathbb{Z}_{(p)}, & \text { if } j=0 \\ p \mathbb{Z}_{(p)}, & \text { if } j=b k, 1 \leq k \leq p-1 \\ \mathbb{Z} / p \mathbb{Z}, & \text { if } j=b k-p^{i}+1,1 \leq k \leq p-1,1 \leq i \leq n-1 \\ 0, & \text { otherwise. }\end{cases}
$$

Proof. We induct on $j=0,1, \ldots, d$. First suppose that $j<b$. The triangle (RM.5) yields an isomorphism

$$
\mathrm{CH}^{j}(\mathcal{R}) \simeq \mathrm{CH}^{j}(\mathcal{X})
$$

and the statement follows from Lemma RM.8.

Now consider the case $j=b$. The triangle (RM.5) yields an exact sequence

$$
\mathrm{CH}^{b}(\mathcal{X}) \rightarrow \mathrm{CH}^{b}(\mathcal{R}) \rightarrow \mathrm{CH}^{0}(\mathcal{S}) \rightarrow H^{2 b+1, b}\left(\mathcal{X}, \mathbb{Z}_{(p)}\right) .
$$

The first term is trivial by Lemma $\mathbb{R M} .8$ and the last is equal to $(\mathbb{Z} / p \mathbb{Z}) \mu$ by Lemma RM.7. By Lemma RM.9, $\mathrm{CH}^{0}(\mathcal{S})=\mathrm{CH}^{0}(\mathcal{R})=\mathbb{Z}_{(p)}$. The last map is the multiplication by $(p-1) \mu=-\mu$ by Lemma RM.3, hence $\mathrm{CH}^{b}(\mathcal{R})=p \mathbb{Z}_{(p)}$.

Now assume that $j=b+1$. The triangle (RM.5) gives an exact sequence

$$
H^{1,1}\left(\mathcal{S}, \mathbb{Z}_{(p)}\right) \rightarrow \mathrm{CH}^{b+1}(\mathcal{X}) \rightarrow \mathrm{CH}^{b+1}(\mathcal{R}) \rightarrow \mathrm{CH}^{1}(\mathcal{S}) .
$$


By Lemma RM.9, the last term is trivial and the first term is equal to

$$
H^{1,1}\left(\mathcal{R}, \mathbb{Z}_{(p)}\right) \simeq H^{1,1}\left(\mathcal{X}, \mathbb{Z}_{(p)}\right) \simeq K_{1}(F) \otimes \mathbb{Z}_{(p)} .
$$

In view of Lemma RM.8, the second term is equal to $K_{1}^{s}(F) \mu$. The first map is multiplication by $\mu$, hence is surjective. It follows that $\mathrm{CH}^{b+1}(\mathcal{R})=0$.

Now suppose that $j>b+1$. The triangle (RM.5) gives an exact sequence

$$
\mathrm{CH}^{j}(\mathcal{X}) \rightarrow \mathrm{CH}^{j}(\mathcal{R}) \rightarrow \mathrm{CH}^{j-b}(\mathcal{S}) \rightarrow H^{2 j+1, j}\left(\mathcal{X}, \mathbb{Z}_{(p)}\right) .
$$

It follows from Lemma RM.9 that $\mathrm{CH}^{j-b}(\mathcal{S}) \simeq \mathrm{CH}^{j-b}(\mathcal{R})$. By Lemmas RM.7 and RM.8, the first and the last terms are trivial, hence

$$
\mathrm{CH}^{j}(\mathcal{R}) \simeq \mathrm{CH}^{j-b}(\mathcal{R})
$$

and the result follows by induction.

\section{Appendix SC. Special CORRESPOndenCeS}

In this Appendix, $\mathrm{CH}$ is the Chow group with integer coefficients and $\mathrm{Ch}$ is the Chow group with coefficients in $\mathbb{F}_{p}$. The base field $F$ is of arbitrary characteristic $\neq p$.

Let $X$ be a smooth complete geometrically irreducible variety of dimension $d:=p^{n}-1$ for some $n \geq 1$. A special correspondence $\sigma$ on $X$ is an anti-symmetric $\left(\sigma^{t}=-\sigma\right)$ element of $\mathrm{CH}^{b}(X \times X)$, where $b=\left(p^{n}-1\right) /(p-1)$, such that for the image $H \in \mathrm{CH}^{b}\left(X_{F(X)}\right)$ of $\sigma$ under the pull-back along the morphism $X_{F(X)} \rightarrow X \times X$, induced by the generic point of the first factor, one has:

(1) $\sigma_{F(X)}=1 \times H-H \times 1$ and

(2) the degree of the 0 -cycle class $H^{p-1}$ is not divisible by $p$.

(The original definition of a special correspondence given in [26] is more restrictive, but we only need the above properties in our business.)

As shown in [26], any standard norm variety possesses a special correspondence, and this explains our interest to varieties possessing a special correspondence.

We are going to use the Steenrod operations on Ch, [4] or [1] (or [2]). For any $i \in \mathbb{Z}$, we write $S^{i}$ for the cohomological Steenrod operation which increases the codimension by $i$. This indexation differs from that of [4]. In our indexation we have $S^{i}=0$ if $i$ is not divisible by $p-1$. Note that for existence of the Steenrod operations, we do not need to assume quasi-projectivity of varieties, [4, §10].

SC-I. Rationality of Steenrod operations. Here is the main result of this subsection which we prove by technique of [8]. It extends (a weakened version of) Theorem 4.3: for $s=0$ the result below is very close to Theorem 4.3 (for $\Lambda=\mathbb{F}_{p}$ ) weakened by the presence of an exponent $p$ element in the statement as well as by the requirement that $Y$ is smooth (which we need for the Steenrod operations to be defined). Note that unlike Theorem 4.3, the proof of the result below does not rely on Appendix RM.

We recall that two smooth complete irreducible varieties are equivalent if there exist multiplicity 1 correspondences (with $\Lambda=\mathbb{F}_{p}$ ) between them in both directions.

Theorem SC.1. Let $X$ be an A-trivial (for $\Lambda=\mathbb{F}_{p}$ ) F-variety equivalent to an A-trivial $F$-variety of dimension $p^{n}-1$ possessing a special correspondence. Then for any smooth irreducible $F$-variety $Y$, any $m, s \in \mathbb{Z}$ with $s>(m-b)(p-1)$, and any $y \in \mathrm{Ch}^{m}\left(Y_{F(X)}\right)$, 
the element $S^{s}(y) \in \mathrm{Ch}^{m+s}\left(Y_{F(X)}\right)$ is rational (i.e., comes from $F$ ) up to the class modulo $p$ of an exponent $p$ element of $\mathrm{CH}^{m+s}\left(Y_{F(X)}\right)$.

Example SC.2. Let $X$ be the Severi-Brauer variety of a degree $p$ central simple $F$ algebra. The variety $X$ has dimension $p-1$, is $A$-trivial (see Example 2.5), and possesses a special correspondence (see [14, Remark 7.17]). It follows that for any smooth $F$-variety $Y$ and any element $y \in \operatorname{Ch}\left(Y_{F(X)}\right)$, its $p$ th power $y^{p}$ is rational up to the class modulo $p$ of an exponent $p$ element. Indeed, one may assume that $Y$ is irreducible and $y$ is homogeneous of some codimension $m \geq 0$. Then $y^{p}=S^{s}(y)$ with $s=m(p-1)>(m-b)(p-1)$ (note that $n=1$ and $b=\left(p^{n}-1\right) /(p-1)=1$ here so that Theorems 4.5 and 4.3, if applicable at all, are vacuous in this situation).

Proof of Theorem $S C$.1. If $\operatorname{deg} \mathrm{CH}_{0}(X) \not \subset p \mathbb{Z}$, then $1 \in \operatorname{deg} \mathrm{Ch}_{0}(X)$ and we are done by Lemma 2.9. Below we are assuming that $\operatorname{deg} \mathrm{CH}_{0}(X) \subset p \mathbb{Z}$.

If the conclusion of Theorem SC.1 holds for an $A$-trivial variety $X$, then it also holds for any $A$-trivial variety $X^{\prime}$ equivalent to $X$. Indeed, by Lemma 2.9, the right and the bottom maps of the commutative square

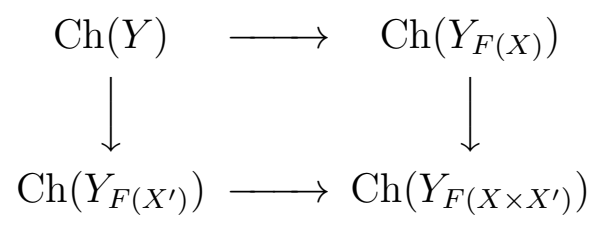

are isomorphisms. Therefore we may assume that the variety $X$ itself has dimension $d:=p^{n}-1$ and possesses a special correspondence $\sigma \in \mathrm{CH}^{b}(X \times X)$. As in our definition of special correspondence, let $H \in \mathrm{CH}^{b}\left(X_{F(X)}\right)$ be the image of $\sigma$.

Lemma SC.3. The element $p H \in \mathrm{CH}^{b}\left(X_{F(X)}\right) \otimes \mathbb{Z}_{(p)}$ is rational.

Proof. We set $\rho:=\sigma^{p-1}$ (the power is taken using multiplication in the Chow group, not composition of correspondences). Then

$$
(\rho \circ \sigma)_{F(X)} / \operatorname{deg}\left(H^{p-1}\right)=1 \times H+(p-1) H \times 1,
$$

and the pull-back of the rational element (SC.4) with respect to the diagonal of $X$ produces $p H$.

The following lemma holds with coefficients in $\mathbb{Z}_{(p)}$ (cf. [26, Proposition 5.9]), although we need it now only for coefficients in $\mathbb{F}_{p}$ :

Lemma SC.5. An abstract Rost motive (with coefficients in $\mathbb{Z}_{(p)}$ as well as with coefficients in $\mathbb{F}_{p}$ ) lives on $X$. More precisely, there exists a symmetric (Rost) projector in $\mathrm{CH}^{d}(X \times X) \otimes \mathbb{Z}_{(p)}$ such that over $F(X)$ it is equal to

$$
\left(1 \times H^{p-1}+H \times H^{p-2}+\cdots+H^{p-1} \times 1\right) / \operatorname{deg}\left(H^{p-1}\right) .
$$

Proof. It suffices to prove the statement for coefficients in $\mathbb{Z}_{(p)}$.

The (symmetric for $p \neq 2$ and anti-symmetric for $p=2$ ) correspondence

$$
\rho:=\sigma^{p-1} \in \mathrm{CH}^{d}(X \times X)
$$

considered over $F(X)$ is congruent modulo $p$ to the alternating sum

$$
1 \times H^{p-1}-H \times H^{p-2}+\cdots+(-H)^{p-2} \times H+(-H)^{p-1} \times 1 .
$$


Let $c \in \mathbb{Z}_{(p)}$ be the inverse of the integer $\operatorname{deg}\left(H^{p-1}\right)$. The symmetric correspondence

$$
\rho^{\prime}:=(c \rho) \circ(c \rho) \in \mathrm{CH}^{d}(X \times X) \otimes \mathbb{Z}_{(p)}
$$

over $F(X)$ is congruent modulo $p$ to the projector

$$
\pi:=c\left(1 \times H^{p-1}+H \times H^{p-2}+\cdots+H^{p-1} \times 1\right) \in \mathrm{CH}^{d}(X \times X)_{F(X)} \otimes \mathbb{Z}_{(p)} .
$$

The motive defined by this projector is

$$
\left(X_{F(X)}, \pi\right) \simeq \mathbb{Z}_{(p)} \oplus \mathbb{Z}_{(p)}(b) \oplus \cdots \oplus \mathbb{Z}_{(p)}(d) .
$$

Replacing $\rho^{\prime}$ by $\left(\rho^{\prime}\right)^{\circ p^{r}}$ with sufficiently big $r$, we keep the symmetry of $\rho^{\prime}$ and get that $\left(\rho^{\prime}\right)_{F(X)} \equiv \pi\left(\bmod p^{p-1}\right)$. It follows by Lemma SC.3 that there exists a symmetric correspondence $\rho^{\prime \prime} \in \mathrm{CH}^{d}(X \times X) \otimes \mathbb{Z}_{(p)}$ with $\rho_{F(X)}^{\prime \prime}=\pi$.

Let $A$ (respectively, $B$ ) be the (commutative) subring of the ring End $M(X)$ (respectively, End $M\left(X_{F(X)}\right)$ ) generated by $\rho^{\prime \prime}$ (respectively, $\pi$ ). The kernel of the ring epimorphism $A \rightarrow B$ consists of nilpotent elements. Indeed, any element of the kernel vanishes over $F(X)$ and, by specialization, over the residue field of any point of $X$. Therefore it is nilpotent by [7, Theorem 67.1]. It follows by an argument like in [7, Corollary 92.5] that there exists a projector in $A \subset \mathrm{CH}^{d}(X \times X) \otimes \mathbb{Z}_{(p)}$ whose image in $B$ is $\pi$. This projector is symmetric because $A$ consists of symmetric elements only. The motive given by this projector is an abstract Rost motive.

Recall that $\operatorname{deg} \mathrm{CH}_{0}(X) \subset p \mathbb{Z}$ is assumed.

Corollary SC.7. For any $i>0$ and any $\alpha \in \mathrm{CH}^{i}(X), \beta \in \mathrm{CH}_{i}\left(X_{F(X)}\right)$, the degree of the 0 -cycle class $\alpha_{F(X)} \cdot \beta$ is divisible by $p$.

Proof. Combine Lemma SC.5 with Corollary 3.11.

Corollary SC.8. For any $i, j>0, k, l \geq 0, \alpha \in \mathrm{CH}^{i}(X)$ and $\beta \in \mathrm{CH}^{j}(X)$, the degree of the element $(\alpha \times \beta)_{F(X)} \cdot\left(H^{k} \times H^{l}\right) \in \mathrm{CH}^{i+j+b(k+l)}(X \times X)_{F(X)}$ is divisible by $p^{2}$.

Proof. The degree is equal to the product of the degrees $\operatorname{deg}\left(\alpha_{F(X)} \cdot H^{k}\right)$ and $\operatorname{deg}\left(\beta_{F(X)} \cdot H^{l}\right)$ each of which is divisible by $p$ by Corollary SC.7.

Corollary SC.9. For any $i, j>0, r \geq 0, \alpha \in \mathrm{CH}^{i}(X)$ and $\beta \in \mathrm{CH}^{j}(X)$, the degree of the element $(\alpha \times \beta) \cdot \sigma^{r} \in \mathrm{CH}^{i+j+b r}(X \times X)$ is divisible by $p^{2}$.

Proof. The element $\sigma_{F(X)}^{r}$ is a linear combination of $H^{k} \times H^{l}($ with $k+l=r)$.

Lemma SC.10. For any $i>0$, the element $S^{i}(H) \in \mathrm{Ch}^{b+i}\left(X_{F(X)}\right)$ is rational.

Proof. We prove first that $\operatorname{deg}\left(H^{j} S^{i}(H)\right) \equiv 0(\bmod p)$ for any $j \geq 0$. Assume the contrary. Then $i$ is divisible by $b$, say, $i=b k$ for some $k>0$, and $j=p-2-k$. Computing the composition $S^{i}(\sigma) \circ \sigma^{p-1-k} \in \mathrm{Ch}(X \times X)$ over $F(X)$ we get a multiple with a nonzero coefficient $\in \mathbb{F}_{p}$ of $H \times 1 \in \mathrm{Ch}^{b}(X \times X)$. Taking the pull-back with respect to the diagonal shows that the class of $H$ modulo $p$ is rational and therefore $1 \in \operatorname{deg} \operatorname{Ch}_{0}(X)$, a contradiction.

Now the composition $S^{i}(\sigma) \circ \sigma^{p-1} \in \mathrm{Ch}(X \times X)$ computed over $F(X)$ gives a multiple with a nonzero coefficient of $1 \times S^{i}(H)$, and we finish the proof pulling back with respect to the diagonal of $X$. 
We set $\rho:=\sigma^{p-1} \in \mathrm{CH}^{d}(X \times X)$. Since $\rho_{F(X)}$ is congruent modulo $p$ to the alternating sum (SC.6), any element $x \in \operatorname{Ch}(X \times Y)$ of the form $x=x^{\prime} \circ \rho$ for some $x^{\prime} \in \operatorname{Ch}(X \times Y)$ decomposes over $F(X)$ as

$$
x_{F(X)}=1 \times x_{0}+H \times x_{1}+\cdots+H^{p-1} \times x_{p-1}
$$

with some $x_{0}, x_{1}, \ldots, x_{p-1} \in \operatorname{Ch}\left(Y_{F(X)}\right)$. Note that $x_{0}$ coincides with the image of $x$ in $\mathrm{Ch}\left(Y_{F(X)}\right)$. The key statement in the proof of Theorem SC.1 is the following Proposition (where the $A$-triviality assumption is not needed):

Proposition SC.12. Let $X$ be a smooth complete irreducible variety of dimension $d=$ $p^{n}-1$ possessing a special correspondence $\sigma \in \mathrm{CH}^{b}(X \times X)$. Let $Y$ be a smooth irreducible variety and $x \in \mathrm{Ch}^{m}(X \times Y)$ an element of the form $x=x^{\prime} \circ \rho$. Then for any $s>$ $(m-b)(p-1)$ the element $S^{s}\left(x_{0}\right) \in \mathrm{Ch}^{m+s}\left(Y_{F(X)}\right)$ is rational up to the class modulo $p$ of an exponent $p$ element.

Proof. For any $x \in \operatorname{Ch}(X \times Y)$, we have the relation

$$
p r_{2 *} \sum_{0 \leq i \leq d+s} b_{i} \cdot S^{d+s-i}(x)=S^{d+s}\left(p r_{2 *}(x)\right),
$$

where $b_{i}:=b_{i}\left(-T_{X}\right) \in \mathrm{Ch}^{i}(X)$ (this is rather $b_{i}\left(T_{X}\right)$ in notation of [26]), where $b_{i}(\cdot)$ are the components of the multiplicative Chern class as defined in [1, §6.1]. Note that the product in the expression $b_{i} \cdot S^{d+s-i}(x)$ is the product of the $\operatorname{Ch}(X)$-module $\operatorname{Ch}(X \times Y)$ so that the expression actually means $\left(b_{i} \times Y\right) \cdot S^{d-i}(x)$ (now in the sense of the product in the ring $\operatorname{Ch}(X \times Y))$.

Now we assume that $x \in \mathrm{Ch}^{m}(X \times Y)$ with $m$ such that $s>(m-b)(p-1)$. In this case $p r_{2 *}(x) \in \mathrm{Ch}^{m-d}(Y)$ and $S^{d+s}\left(p r_{2 *}(x)\right)=0$ because $d+s>s>(m-b)(p-1) \geq$ $(m-d)(p-1)$. Besides, $S^{d+s}(x)=0$ because $d+s=b(p-1)+s>m(p-1)$. Therefore we have

$$
\operatorname{pr}_{2 *} \sum_{0<i \leq d+s} b_{i} \cdot S^{d+s-i}(x)=0 .
$$

Putting $x \circ \rho$ in place of $x$ in relation (SC.13) and using the equality

$$
S^{\bullet}(x \circ \rho)=\left(b_{\bullet} \cdot S^{\bullet}(x)\right) \circ S^{\bullet}(\rho),
$$

(together with the projection formula) we rewrite the left part of relation (SC.13) as

$$
\begin{aligned}
p r_{2 *} \sum_{\substack{i+j+k+l=d+c \\
i>0, j, k, l \geq 0}} b_{i} \cdot\left(\left(b_{j}\right.\right. & \left.\left.S^{k}(x)\right) \circ S^{l}(\rho)\right) \\
& =p r_{2 *} \sum p r_{13 *}\left(\left(\left(b_{i} \cdot S^{l}(\rho)\right) \times[Y]\right) \cdot\left([X] \times\left(b_{j} \cdot S^{k}(x)\right)\right)\right) \\
& =p r_{2 *} \sum p r_{23 *}\left(\left(\left(b_{i} \cdot S^{l}(\rho)\right) \times[Y]\right) \cdot\left([X] \times\left(b_{j} \cdot S^{k}(x)\right)\right)\right) \\
& =p r_{2 *} \sum\left(b_{j} \cdot S^{k}(x)\right) \cdot\left(\left(p r_{2 *}\left(b_{i} \cdot S^{l}(\rho)\right)\right) \times[Y]\right) \\
& =p r_{2 *} \sum b_{j} \cdot\left(p r_{2 *}\left(b_{i} \cdot S^{l}(\rho)\right)\right) \cdot S^{k}(x) .
\end{aligned}
$$


Therefore

$$
p r_{2 *} \sum_{\substack{i+j+k+l=d+s \\ i>0, j, k, l \geq 0}} b_{j} \cdot\left(p r_{2 *}\left(b_{i} \cdot S^{l}(\rho)\right)\right) \cdot S^{k}(x)=0
$$

for any $x \in \mathrm{Ch}^{m}(X \times Y)$.

We recall that $\rho=\sigma^{p-1}$. Therefore

$$
S^{l}(\rho)=S^{l}\left(\sigma^{p-1}\right)=\sum_{l_{1}+\cdots+l_{p-1}=l} S^{l_{1}}(\sigma) \cdot \ldots \cdot S^{l_{p-1}}(\sigma)
$$

Relation (SC.14) rewrites as

$$
p r_{2 *} \sum_{\substack{i+j+k+l_{1}+\cdots+l_{p-1}=d+s \\ i>0 ; j, k, l_{1}, \ldots, l_{p-1} \geq 0}} b_{j} \cdot\left(p r_{2 *}\left(b_{i} \cdot S^{l_{1}}(\sigma) \cdot \ldots \cdot S^{l_{p-1}}(\sigma)\right)\right) \cdot S^{k}(x)=0 .
$$

Therefore, fixing for each integer $k \geq 0$ an integral representative $S_{x}^{k} \in \mathrm{CH}^{m+k}(X \times Y)$ of $S^{k}(x) \in \mathrm{Ch}^{m+k}(X \times Y)$ as well as an integral representative $S_{\sigma}^{k} \in \mathrm{CH}^{b+k}(X \times X)$ of $S^{k}(\sigma) \in \mathrm{Ch}^{b+k}(X \times X)$ (where we choose $\sigma$ for $S_{\sigma}^{0}$ ), we get that the sum

$$
\sum_{\substack{i+j+k+l_{1}+\cdots+l_{p-1}=d+s \\ i>0 ; j, k, l_{1}, \ldots, l_{p-1} \geq 0}} p r_{2 *}\left(b_{j} \cdot\left(p r_{2 *}\left(b_{i} \cdot S_{\sigma}^{l_{1}} \cdot \ldots \cdot S_{\sigma}^{l_{p-1}}\right)\right) \cdot S_{x}^{k}\right)
$$

is divisible by $p$ in $\mathrm{CH}(Y)$. Taking for $x$ an element of the form $x=x^{\prime} \circ \rho$ with some $x^{\prime} \in \mathrm{Ch}^{m}(X \times Y)$ and passing over $F(X)$, we are going to show that the sum of (SC.15) is equal modulo $I:=p^{2} \mathrm{CH}\left(Y_{F(X)}\right)+p \operatorname{Im}\left(\mathrm{CH}(Y) \rightarrow \mathrm{CH}\left(Y_{F(X)}\right)\right)$ to the class of $\operatorname{deg}\left(b_{d}\right) S_{x_{0}}^{s}$, where $S_{x_{0}}^{s} \in \mathrm{Ch}^{m+s}\left(Y_{F(X)}\right)$ is an integral representative of $S^{s}\left(x_{0}\right)$. More precisely, we show that the summand for $i=d$ and $k=s$ modulo $I$ is $\operatorname{deg}\left(b_{d}\right) S_{x_{0}}^{s}$ (up to multiplication by a prime to $p$ integer) while each other summand modulo $I$ is 0 . Since the integer $\operatorname{deg} b_{d}$ is not divisible by $p^{2}$ (see [26, Theorem 9.9]), we will get that $S_{x_{0}}^{s}$ is rational up to the classes modulo $p$ of an element of exponent $p$.

For $i=d$ and $k=s$ we have $j=l_{1}=\cdots=l_{p-1}=0$, and the corresponding summand of (SC.15) is equal to

$$
p r_{2 *}\left(p r_{2 *}\left(b_{d} \cdot \rho\right) \cdot S_{x}^{s}\right)_{F(X)} .
$$

Since $\operatorname{deg}\left(b_{d}\right)$ is divisible by $p$ and $\rho_{F(X)}$ is congruent modulo $p$ to (SC.6), the factor $p r_{2 *}\left(b_{d} \cdot \rho\right)$ is congruent modulo $I$ to $\operatorname{deg}\left(b_{d}\right) \cdot H^{p-1}$. Taking into account the decomposition (SC.11) of $x_{F(X)}$, it follows that (SC.16) is congruent modulo $I$ to $\operatorname{deg}\left(b_{d}\right) S_{x_{0}}^{s}$ up to multiplication by the prime to $p$ integer $\operatorname{deg}\left(H^{p-1}\right)$. Below we are assuming that $i \neq d$.

For $l=0$ (where $l:=l_{1}+\cdots+l_{p-1}$ ), that is to say, for $l_{1}=\cdots=l_{p-1}=0$, an arbitrary summand we get is of the form

$$
p r_{2 *}\left(b_{j} \cdot p r_{2 *}\left(b_{i} \cdot \rho\right) \cdot S_{x}^{k}\right)_{F(X)}
$$

(with $i+j+k=d+s$ ). Note that $p r_{2 *}\left(b_{i} \cdot \rho\right)_{F(X)}=0$ if $i$ is not divisible by $b$. Otherwise, since $i>0$ and $\rho_{F(X)}$ is congruent modulo $p$ to (SC.6), $p r_{2 *}\left(b_{i} \cdot \rho\right)_{F(X)}$ is by Corollary 
SC.7 congruent modulo $I$ to a multiple of $p H^{i / b}$ so that we only need to show that the element

$$
\operatorname{pr}_{2 *}\left(b_{j} \cdot H^{i / b} \cdot S^{k}(x)\right)_{F(X)} \in \operatorname{Ch}\left(Y_{F(X)}\right)
$$

is rational.

If $j>0$, then computing $S^{k}(x)_{F(X)}=S^{k}\left(x_{F(X)}\right)$ via the decomposition (SC.11) of $x_{F(X)}$ and using Corollary SC.7, we see that the element (SC.17) is 0. Let us assume that $j=0$ and show that (SC.17) is 0 as well. It suffices to show this with $x$ replaced by an arbitrary summand of the decomposition (SC.11). Putting $1 \times x_{0}$ (the first summand of the decomposition) in place of $x$, we get

$$
p r_{2 *}\left(H^{i / b} \cdot S^{k}\left(1 \times x_{0}\right)\right)=p r_{2 *}\left(H^{i / b} \times S^{k}\left(x_{0}\right)\right)
$$

which is 0 because $i \neq d$. Putting any other summand $H^{r} \times x_{r}(r \geq 1)$ of the decomposition, we get a multiple of $S^{s+r b}\left(x_{r}\right)$ which is 0 because $x_{r} \in \mathrm{Ch}^{m-r b}\left(Y_{F(X)}\right)$ and $s+r b>s>(m-b)(p-1) \geq(m-r b)(p-1)$.

It remains to consider the case of $l>0$. We have

$$
S^{l}(\sigma)_{F(X)}=1 \times S^{l}(H)-S^{l}(H) \times 1
$$

and $S^{l}(H)$ is rational by Lemma SC.10. Therefore

$$
\left(S_{\sigma}^{l}\right)_{F(X)}=p \theta_{l}+1 \times S_{H}^{l}-S_{H}^{l} \times 1
$$

for some $\theta_{l} \in \mathrm{CH}^{b+l}(X \times X)_{F(X)}$ and a rational integral representative $S_{H}^{l}$ of $S^{l}(H)$.

Let us decompose as in (SC.18) every factor with positive superscript of the product

$$
\left(S_{\sigma}^{l_{1}} \cdot \ldots \cdot S_{\sigma}^{l_{p-1}}\right)_{F(X)}
$$

(appearing in $(\text { SC.15 })_{F(X)}$ ), expand the product and consider an arbitrary summand $P$ of the expansion. We are going to show that the element

$$
p r_{2 *}\left(b_{j} \cdot p r_{2 *}\left(b_{i} \cdot P\right) \cdot\left(S_{x}^{k}\right)_{F(X)}\right)
$$

modulo $I$ is 0 .

If $P$ contains the factor $p \theta_{\text {? }}$ (at least) two times, then the result is divisible by $p^{2}$ so that (SC.19) is indeed 0 modulo $I$.

Assume that the factor $p \theta_{\text {? }}$ is present precisely one time in $P$. So, we already have divisibility by $p$ and it suffices to show that the element

$$
p r_{2 *}\left(t \cdot S^{k}\left(x_{F(X)}\right)\right) \in \mathrm{Ch}^{m+s}\left(Y_{F(X)}\right)
$$

is 0 for an element $t \in \mathrm{Ch}^{i+j+l+d}\left(X_{F(X)}\right)$ such that $p \cdot t=b_{j} \cdot p r_{2 *}\left(b_{i} \cdot P\right)$. Replacing $x_{F(X)}$ in (SC.20) by an arbitrary summand of the decomposition (SC.11), we get 0 always (and for an arbitrary $\left.t \in \mathrm{Ch}^{i+j+l+d}\left(X_{F(X)}\right)\right)$ with only one possible exception: for the summand $1 \times x_{0}$, namely.

Putting $1 \times x_{0}$ in place of $x_{F(X)}$ in (SC.20), we may get a nonzero result only if $k=s$, that is, $i+j+l=d$. In this case $t$ is a 0-cycle class and the element (SC.20) is divisible by its degree. It suffices therefore to show that the degree $\operatorname{deg}\left(b_{j} \cdot p r_{2 *}\left(b_{i} \cdot P\right)\right)$ is divisible by $p^{2}$. If $j>0$, then the degree is divisible by $p^{2}$ by Corollary SC.7 (recall that $P$ is divisible by $p$ ). Therefore we may assume that $j=0$, that is $i+l=d$. In this case $b_{i} \cdot P$ 
is a 0-cycle class (on $X \times X)$ and the corresponding summand of (SC.15) is divisible by its degree. But degree of $b_{i} \cdot P$ coincides with degree of $b_{i} \cdot p r_{1 *}(P)$ which is divisible by $p^{2}$ by Corollary SC.7 (we recall that $i>0$ and that $P$ is already divisible by $p$ ).

At last, let us assume that $P$ contains no $\theta_{\text {? }}$ as a factor. Then $P$ must contain at least one factor of the type $1 \times S_{H}^{?}$ (we call it a second type factor because it corresponds to the second summand of the decomposition (SC.18)) or of the type $S_{H}^{?} \times 1$ (a third type factor). Moreover, any factor of $P$ is either $\sigma$ or $1 \times S_{H}^{?}$ (a second type factor) or $S_{H}^{?} \times 1$ (a third type factor). It follows by Corollary SC.7 that $p r_{2 *}\left(b_{i} \cdot P\right)$ is divisible by $p$. Therefore we may assume that $k=s$. The element (SC.19) is then divisible by degree of the 0 -cycle class $\left(b_{i} \times b_{j}\right) \cdot P$. This degree is divisible by $p^{2}$ if $j>0$ or if $P$ contains a factor of the second type by Corollary SC.9.

In the remaining case we have $j=0, i+l=d$, any factor of $P$ equals $\sigma$ or has the third type with at least one factor of the third type. Therefore $P=(\alpha \times 1) \cdot \sigma^{r}$ with some $r<p-1$ and some $\alpha \in \mathrm{CH}\left(X_{F(X)}\right)$ (which is in fact rational but we do not care about this anymore). It follows that the element $\operatorname{pr}_{2 *}\left(b_{i} \cdot P\right)$ is a 0 -cycle class and the element (SC.19) is divisible by its degree which is

$$
\operatorname{deg}\left(p r_{2 *}\left(b_{i} \cdot P\right)\right)=\operatorname{deg}\left(p r_{1 *}\left(b_{i} \cdot P\right)\right)=\operatorname{deg}\left(b_{i} \cdot p r_{1 *}(P)\right),
$$

but already the element

$$
p r_{1 *}(P)=\alpha \cdot p r_{1 *}\left(\sigma^{r}\right)
$$

is trivial because $p r_{1 *}\left(\sigma^{r}\right)=0$ for $r<p-1$.

We finish now the proof of Theorem SC.1. Let $x$ be an element of $\mathrm{Ch}^{m}(X \times Y)$ mapped to $y \in \mathrm{Ch}^{m}\left(Y_{F(X)}\right)$. Since $X$ is $A$-trivial, the element $(c x) \circ \rho$, where $c \in \mathbb{F}_{p}$ is the inverse to the class modulo $p$ of the integer $\operatorname{deg}\left(H^{p-1}\right)$, is also mapped to $y$ (see Lemma 2.6). Replacing $x$ by $(c x) \circ \rho$, we apply Proposition SC.12 to the new $x$ getting the desired result.

SC-II. Generators of Chow groups of Rost motives. In this appendix, we provide an elementary construction of homogeneous generators of the Chow group of a Rost motive in the spirit of [12]. Here we have $\Lambda=\mathbb{Z}_{(p)}$ so that $\mathrm{CH}$ stands for the Chow group with coefficients in $\mathbb{Z}_{(p)}$.

Let $X$ be a standard norm variety of dimension $d:=p^{n}-1$ ( $p$ a prime, $\left.n \geq 1\right)$. Let $\sigma \in \mathrm{CH}^{b}(X \times X), b:=d /(p-1)$, be a special correspondence on $X, H \in \mathrm{CH}^{b}\left(X_{F(X)}\right)$ the image of $\sigma$. Let $\rho \in \mathrm{CH}^{d}(X \times X)$ be a symmetric (Rost) projector on $X$ such that

$$
\rho_{F(X)}=\left(1 \times H^{p-1}+H \times H^{p-2}+\cdots+H^{p-1} \times 1\right) / \operatorname{deg}\left(H^{p-1}\right)
$$

(see Lemma SC.5).

Let $m$ be an integer satisfying $1 \leq m \leq n-1$. Assume that there exists a norm variety $Y$ of dimension $p^{m}-1$ with a morphism $f: Y \rightarrow X$. (This assumption is satisfied if the base field $F$ is $p$-special and has characteristic 0 by [28, Corollary 1.22].)

Proposition SC.21. For any $r$ with $1 \leq r \leq p-1$, the element

$$
\alpha:=\rho_{*}\left(\sigma^{r}\right)_{*} f_{*}[Y] \in \mathrm{CH}_{p^{m}-1+(p-1-r) b}(X)
$$

is of order $p$. 
Remark SC.22. Since $\rho$ is symmetric, we have $\rho_{*}=\rho^{*}$ and therefore

$$
\alpha \in \rho^{*} \mathrm{CH}^{p^{m}-1+(p-1-r) b}(X)=\mathrm{CH}^{p^{m}-1+(p-1-r) b}(X, \rho) .
$$

Since the Chow group is of order $p$ by Theorem RM.10, $\alpha$ is its generator. By Theorem RM.10once again, varying $m$ and $r$, we get generators for the whole torsion part of the Chow group $\mathrm{CH}^{*}(X, \rho)$ of the Rost motive.

Proof of Proposition SC.21. We first check that $p \alpha=0$. Since $X$ has a closed point of degree $p$, it suffices to check that $\alpha_{F(X)}=0$. Over $F(X)$ we have

$$
\alpha_{F(X)}=(\beta)_{*} f_{*}[Y]
$$

where $\beta$ is a linear combination of $H^{i} \times H^{r-i}$ for $i=0,1, \ldots, r$. This is 0 because $\operatorname{dim} Y \neq \operatorname{codim} H^{i}=b i$ for any $i$. Indeed,

$$
0<\operatorname{dim} Y<p^{n-1} \leq 1+p+\cdots+p^{n-1}=b .
$$

Now we show that $\alpha \neq 0$ as follows:

$$
\alpha \neq 0 \Leftarrow \alpha \bmod p \neq 0 \Leftarrow S^{p^{m}-1}(\alpha) \neq 0 \Leftarrow p^{2} \backslash \operatorname{deg}\left(\left(S_{\alpha}^{p^{m}-1}\right)_{F(X)} \cdot H^{p-1-r}\right),
$$

where $S_{\alpha}^{p^{m}-1} \in \mathrm{CH}_{(p-1-r) b}(X)$ is a representative of the modulo $p$ cycle class $S^{p^{m}-1}(\alpha) \in$ $\mathrm{Ch}_{(p-1-r) b}(X)$. The implication on the very right comes from Corollary SC.7. (The degree modulo $p^{2}$ does not depend on the choice of the integral representative by Corollary SC.7 once again.)

In order to compute $\operatorname{deg}\left(\left(S_{\alpha}^{p^{m}-1}\right)_{F(X)} \cdot H^{p-1-r}\right)$ we use the formula

$$
S^{\bullet}\left(\left(\rho \circ \sigma^{r}\right)_{*} f_{*}[Y]\right)=\left(S^{\bullet}\left(\rho \circ \sigma^{r}\right)\right)_{*} S_{\bullet} f_{*}[Y],
$$

where $S_{\bullet}$ is the total homological Steenrod operation. Since $S_{\bullet} f_{*}[Y]=f_{*}\left(S_{\bullet}[Y]\right)=b_{\bullet}^{Y}$, where $b_{\bullet}^{Y}:=f_{*}\left(b_{\bullet}\left(-T_{Y}\right)\right)$, the degree in question is congruent modulo $p^{2}$ to

$$
\begin{aligned}
\operatorname{deg} \sum_{\substack{i+j=p^{m}-1 \\
i, j \geq 0}} H^{p-1-r} \cdot\left(S_{\rho \circ \sigma^{r}}^{j}\right)_{*}\left(b_{i}^{Y}\right)_{F(X)} & \\
& =\sum \operatorname{deg}\left(H^{p-1-r} \cdot p r_{2 *}\left(b_{i}^{Y} \cdot S_{\rho \circ \sigma^{r}}^{j}\right)_{F(X)}\right) \\
& =\sum \operatorname{deg}\left(\left(\left(b_{i}^{Y}\right)_{F(X)} \times H^{p-1-r}\right) \cdot\left(S_{\rho \circ \sigma^{r}}^{j}\right)_{F(X)}\right) \\
& =\sum \operatorname{deg}\left(\left(b_{i}^{Y}\right)_{F(X)} \cdot p r_{1 *}\left(\left(S_{\rho \circ \sigma^{r}}^{j}\right)_{F(X)} \cdot\left(1 \times H^{p-1-r}\right)\right)\right),
\end{aligned}
$$

where $S_{\rho \circ \sigma^{r}}^{j}$ are representatives of $S^{j}\left(\rho \circ \sigma^{r}\right)$. Let us choose representatives $S_{\sigma^{r}}^{j}$ of $S^{j}\left(\sigma^{r}\right)$. Since $\left(\rho \circ \sigma^{r}\right)_{F(X)}=\sigma_{F(X)}^{r}$, the classes modulo $p$ of $\left(S_{\rho \circ \sigma^{r}}^{j}\right)_{F(X)}$ and $\left(S_{\sigma^{r}}^{j}\right)_{F(X)}$ coincide. It follows by Corollary SC.7 that we may remove $\rho$ from the formula (the resulting degree modulo $p^{2}$ is not changed).

Taking $j=0$, we get the product $\operatorname{deg} b_{\operatorname{dim} Y}^{Y} \cdot \operatorname{deg}\left(H^{p-1}\right)$ which is not divisible by $p^{2}$. It remains to show that for any $j>0$ the degree

$$
\operatorname{deg}\left(\left(b_{i}^{Y} \times H^{p-1-r}\right) \cdot S_{\sigma^{r}}^{j}\right)=\operatorname{deg}\left(b_{i}^{Y} \cdot p r_{1 *}\left(S_{\sigma^{r}}^{j} \cdot\left(1 \times H^{p-1-r}\right)\right)\right)
$$


(everything is over $F(X)$ although we omit the subscription $F(X))$ is divisible by $p^{2}$. We have

$$
S\left(\sigma^{r}\right)=\sum_{j_{1}+\cdots+j_{r}=j} S^{j_{1}}(\sigma) \cdot \ldots \cdot S^{j_{r}}(\sigma) .
$$

As in the end of Subsection SC-1, for any $j>0$, we have

$$
S_{\sigma}^{j}=p \theta_{j}+1 \times S_{H}^{j}-S_{H}^{j} \times 1
$$

for some $\theta_{j} \in \mathrm{CH}^{b+j}(X \times X)_{F(X)}$ and a rational integral representative $S_{H}^{j}$ of $S^{j}(H)$.

Let us decompose as in (SC.23) every factor with positive superscript of the product

$$
S_{\sigma}^{j_{1}} \cdot \ldots \cdot S_{\sigma}^{j_{r}}
$$

expand the product and consider an arbitrary summand $P$ of the expansion. We want to show that the degree

$$
\operatorname{deg}\left(\left(b_{i}^{Y} \times H^{p-1-r}\right) \cdot P\right)=\operatorname{deg}\left(b_{i}^{Y} \cdot p r_{1 *}\left(P \cdot\left(1 \times H^{p-1-r}\right)\right)\right)
$$

is divisible by $p^{2}$.

If $P$ contains a factor of the type $p \theta_{\text {? }}$ (at least) one time, then the result is divisible by $p^{2}$ by Corollary SC.7 applied to the right-hand side presentation of the degree in (SC.24).

Let us assume that $P$ contains no $\theta_{\text {? }}$ as a factor. Then $P$ must contain at least one factor of the second or of the third type. Moreover, any factor of $P$ is either $\sigma$ or $1 \times S_{H}^{\text {? }}$ (a second type factor) or $S_{H}^{?} \times 1$ (a third type factor). If a factor of the second type is present, the degree is divisible by $p^{2}$ by Corollary SC.8 applied to the left-hand side presentation of the degree in (SC.24). If there is no factor of the second type, then already $p r_{1 *}\left(P \cdot\left(1 \times H^{p-1-r}\right)\right)=0$ showing that the degree (in its right-hand side presentation) is 0 .

\section{REFERENCES}

[1] Boisvert, A. A new definition of the Steenrod operations in algebraic geometry. arXiv:0805.1414v1 [math.KT] (9 May 2008), 28 pages.

[2] Boisvert, A. R. A new definition of the Steenrod operations in algebraic geometry. ProQuest LLC, Ann Arbor, MI, 2007. Thesis (Ph.D.)-University of California, Los Angeles.

[3] Brosnan, P. A short proof of Rost nilpotence via refined correspondences. Doc. Math. 8 (2003), 69-78.

[4] Brosnan, P. Steenrod operations in Chow theory. Trans. Amer. Math. Soc. 355, 5 (2003), 18691903 (electronic).

[5] Chernousov, V., And Merkurjev, A. Motivic decomposition of projective homogeneous varieties and the Krull-Schmidt theorem. Transform. Groups 11, 3 (2006), 371-386.

[6] Draxl, P. K. Skew fields, vol. 81 of London Mathematical Society Lecture Note Series. Cambridge University Press, Cambridge, 1983.

[7] Elman, R., Karpenko, N., And Merkurjev, A. The algebraic and geometric theory of quadratic forms, vol. 56 of American Mathematical Society Colloquium Publications. American Mathematical Society, Providence, RI, 2008.

[8] Fino, R. Around rationality of cycles. Linear Algebraic Groups and Related Structures (preprint server) 450 (2011, Nov 16), 11 pages.

[9] Fulton, W. Intersection theory, second ed., vol. 2 of Ergebnisse der Mathematik und ihrer Grenzgebiete. 3. Folge. A Series of Modern Surveys in Mathematics [Results in Mathematics and Related Areas. 3rd Series. A Series of Modern Surveys in Mathematics]. Springer-Verlag, Berlin, 1998. 
[10] Garibaldi, S. Cohomological invariants: exceptional groups and spin groups. Mem. Amer. Math. Soc. 200, 937 (2009), xii+81. With an appendix by Detlev W. Hoffmann.

[11] Grothendieck, A. Techniques de construction et théorèmes d'existence en géométrie algébrique. IV. Les schémas de Hilbert. In Séminaire Bourbaki, Vol. 6. Soc. Math. France, Paris, 1995, pp. Exp. No. 221, 249-276.

[12] Karpenko, N., and Merkurjev, A. Rost projectors and Steenrod operations. Doc. Math. 7 (2002), 481-493 (electronic).

[13] Karpenko, N. A. Upper motives of algebraic groups and incompressibility of Severi-Brauer varieties. Linear Algebraic Groups and Related Structures (preprint server) 333 (2009, Apr 3, revised: 2009, Apr 24), 18 pages. J. Reine Angew. Math., to appear.

[14] Karpenko, N. A. Criteria of motivic equivalence for quadratic forms and central simple algebras. Math. Ann. 317, 3 (2000), 585-611.

[15] Karpenko, N. A. Weil transfer of algebraic cycles. Indag. Math. (N.S.) 11, 1 (2000), 73-86.

[16] Karpenko, N. A., And Merkurjev, A. S. Canonical p-dimension of algebraic groups. Adv. Math. 205, 2 (2006), 410-433.

[17] Manin, J. I. Correspondences, motifs and monoidal transformations. Mat. Sb. (N.S.) 77 (119) (1968), 475-507.

[18] Merkurjev, A. Rost invariants of simply connected algebraic groups. In Cohomological invariants in Galois cohomology, vol. 28 of Univ. Lecture Ser. Amer. Math. Soc., Providence, RI, 2003, pp. 101158. With a section by Skip Garibaldi.

[19] Merkurjev, A. Unramified elements in cycle modules. J. Lond. Math. Soc. (2) 78, 1 (2008), 51-64.

[20] Merkurjev, A., And Suslin, A. Motivic cohomology of the simplicial motive of a Rost variety. J. Pure Appl. Algebra 214, 11 (2010), 2017-2026.

[21] Merkurjev, A. S. Essential dimension. In Quadratic Forms - Algebra, Arithmetic, and Geometry, vol. 493 of Contemp. Math. Amer. Math. Soc., Providence, RI, 2009, pp. 299-326.

[22] Merkurjev, A. S., And Suslin, A. A. $K$-cohomology of Severi-Brauer varieties and the norm residue homomorphism. Izv. Akad. Nauk SSSR Ser. Mat. 46, 5 (1982), 1011-1046, 1135-1136.

[23] NguYen, D. H. On $p$-generic splitting varieties for Milnor $K$-symbols mod $p$. arXiv:1003.3971v1 [math.AG] (21 Mar 2010), 19 pages.

[24] NGuyen, D. H. On p-generic splitting varieties for Milnor K-symbols mod p. ProQuest LLC, Ann Arbor, MI, 2009. Thesis (Ph.D.)-University of California, Los Angeles.

[25] PAnin, I. Application of $K$-theory in algebraic geometry. Ph.D. thesis, LOMI, Leningrad, 1984.

[26] Rost, M. On the basic correspondence of a splitting variety. Informal notes, September-November 2006, 42 pages. Available on the web page of the author.

[27] Rost, M. Chow groups with coefficients. Doc. Math. 1 (1996), No. 16, 319-393 (electronic).

[28] Suslin, A., And Joukhovitski, S. Norm varieties. J. Pure Appl. Algebra 206, 1-2 (2006), 245-276.

[29] Thakur, M. L. Isotopy and invariants of Albert algebras. Comment. Math. Helv. 74, 2 (1999), 297-305.

[30] VisHIK, A. Generic points of quadrics and Chow groups. Manuscripta Math. 122, 3 (2007), 365-374.

[31] Vishik, A. Rationality of integral cycles. Doc. Math., Extra volume: Andrei A. Suslin sixtieth birthday (2010), 661-670.

[32] Vishik, A., And Zainoulline, K. Motivic splitting lemma. Doc. Math. 13 (2008), 81-96.

[33] Voevodsky, V. On motivic cohomology with $\mathbb{Z} / l$-coefficients. Ann. of Math. (2) 174, 1 (2011), 401-438.

[34] Zainoulline, K. Special correspondences and Chow traces of Landweber-Novikov operations. $J$. Reine Angew. Math. 628 (2009), 195-204.

Institut de Mathématiques de Jussieu, Université Pierre et Marie Curie, Paris, FRANCE E-mail address: karpenko at math.jussieu.fr

Department of Mathematics, University of California, los Angeles, CA, USA

E-mail address: merkurev at math.ucla.edu 